\title{
Does Shorter Postnatal Hospital Stay lead to Post-discharge Complications? An Instrumental Variables Approach
}

\author{
Mohammad Mahbubur Rahman, Saseendran Pallikadavath, Rebeya Khatoon
}

\begin{abstract}
We exploit the difference in means of postnatal hospital stays between beneficiary mothers of Janani Suraksha Yojana (JSY) and control mothers to estimate causal effects of postnatal hospital stay (PHS) on post-discharge complications (PDCs) of a mother and her newborn. We argue that JSY increases the demand for institutional deliveries in those hospitals, which are assigned by the government to provide such services to the JSY recipients. Given the limited supply of beds, health professionals, and other facilities, an excess demand for institutional deliveries in those hospitals force JSY recipients to stay shorter after births compared to nonrecipients of JSY who are free to deliver in any hospital. Thus, the dummy for JSY becomes a suitable instrument for PHS. Using instrumental variables (IV) regressions, we find that PHS has statistically significant negative causal effects on half of the available PDCs in the data, including abdominal pain, vaginal discharge, convulsion and severe headache of a mother, and fever or cold of the newborn.
\end{abstract}

JEL Classification: C31, C36, I10, I18

Key words: Janani Suraksha Yojana; instrumental variables regressions; postnatal hospital stay; post-discharge complications 


\section{Introduction}

Both a mother and her newborn may experience several complications in the postpartum period. Skilled healthcare professionals can identify some of them immediately after birth and others somewhat late. For example, they may recognize cardiopulmonary problems of a newborn, relating to the transition from an intrauterine to an extra-uterine environment, within 12 hours of birth, as they are apparent at that time, but significant jaundice, ductal-dependent cardiac lesions, gastrointestinal obstruction and other problems can be visible after 12 hours (Desmond et al., 1966; Ip et al., 2004; Gentile et al., 1981; Lambert et al., 1966; Lister et al., 1990; Jackson et al., 2000). In the case of a mother, hemorrhage, followed by excessive bleeding at the time of delivery, and depression (e.g., baby blues, postpartum depression) can be seen within 12 hours of birth. Other complications such as vaginal discharge, difficulty in urinating and fever may happen due to a bacterial infection near the perineal tear or an infection of the uterine lining, and therefore they may take a longer time to be identified.

Postnatal hospital stay (PHS) of a mother and her newborn should be sufficient so that, at the time of stay, almost all possible postnatal complications can be recognized and treated properly by healthcare professionals, and both of them are out of danger, and the family is able and prepared to take care of them at home. Otherwise, there is a chance of re-hospitalization. A shorter stay may even cause premature cessation of breastfeeding, reduce in-hospital teaching on infant care and mother's health, and increase parental anxiety (Arnold and Bernstein, 2000; Britton et al., 1999; Maisels and Kring, 1998; Braveman et al., 1995). There are also arguments for a shorter stay, as it decreases the risk of iatrogenic infection, reduces the hospital cost, improves patient satisfaction, and also promotes family bonding and attachment (Patterson, 1987; Waldenstr" om et al., 1987). In spite of them, an early discharge is still considered as a potential threat to maternal and child health. ${ }^{1}$

However, if a longer PHS does not guarantee a better care, that kind of stay may bias any causal effects on post-discharge complications (PDCs). For example, many bottom level government hospitals in developing countries do not have qualified doctors and nurses, and staying there is unlikely to reduce PDCs. Moreover, the relationships between PHS and PDCs can be nonlinear. A small stay gap may not make any difference in PDCs of those women who stay longer even in a top government hospital or a private hospital. Usually, women with delivery time complications (e.g., C-sections) stay longer. Those who have vaginal deliveries stay relatively shorter, and their small stay gaps may make significant differences in their PDCs. As the relationships between PHSs and PDCs (especially in the developing country context) can be influenced by socioeconomic conditions of women, hospital types, and delivery time complications, estimating authentic causal effects will need a careful handling of them through rigorous econometric analyses.

Over the last five decades, both developed and developing countries have been experiencing declines in averages of PHSs in the cases of both cesarean and vaginal deliveries (Ford et al., 2012;

\footnotetext{
${ }_{1}$ After observing declines in means of PHSs, to increase them the USA changed federal laws in the mid-1990s in most of its states, setting the minimum PHSs at 48 hours for a vaginal delivery and 96 hours for the cesarean delivery (American Academy of Pediatrics and others, 2010). Thus, in the USA, an early discharge is referred to a PHS of $<48$ hours for a vaginal delivery and of $<96$ hours for the cesarean delivery. Both developed and developing countries' studies determined the early discharge in that way. According to WHO (2013) and Campbell et al. (2006), after an uncomplicated vaginal birth in a health facility, both the mother and the newborn should receive care in the facility for at least 24 hours.
} 
Datar and Sood, 2006; DeFrances et al., 2008; Gupta et al., 2006). These have drawn the attention of researchers for about the last three decades. Several studies examined whether the early discharge increases the probability of readmission or chances of PDCs (Datar and Sood, 2006; Madden et al., 2003; Madden et al., 2004; Meara et al., 2004; Kotagal et al., 1999; Watt et al., 2005; Grupp-Phelan et al., 1999; Paul et al., 2006). However, most of the previous studies estimated associations, not causal effects, between early discharge and readmission, by typically running logit regressions with small data. It is likely that an association is an overestimate or underestimate of the causal effect. For example, if an increase in stay leads to infections for some patients, the negative effect of this increase on complications will be less prominent. The estimated effect will be thus upward biased. A similar effect arises due to low-quality postnatal care in the hospitals, and/or households with good family doctors and therefore better care at home. They will prefer them to be discharged earlier from the hospital. Such scenarios are seen in more affluent societies (Kersnik, 2001). On the other hand, an opposite effect may happen if husbands do not allow their wives and newborns to stay longer in a hospital after births for socioeconomic reasons. Moreover, after their discharge, they may receive adverse behavior from those husbands and their family members. Such cases may lead to PDCs, then re-admissions, and thereby a downward bias of the causal effect. ${ }^{2}$ Besides, most of the previous studies estimated an early discharge as a dummy variable based on 48 hours of stay (1 if PHS is less than 48 hours, 0 otherwise). However, this seems quite restrictive as it is unlikely to have a discontinuity in the probability of re-admission at 48 hours. Rather a continuous variable such as hours of PHS seems more relevant in this context.

In this study, we use hours of PHS instead of the early discharge dummy and address the endogeneity of it by running instrumental variables (IV) regressions. We use the dummy for Janani Suraksha Yojana (JSY), one of the most extensive demand-side financing programs in the world providing cash to women for institutional deliveries, as an instrument for hours of PHS. We find that the mean hours of PHS of JSY recipients is lower than that of nonrecipients of JSY with similar socioeconomic characteristics. JSY recipients are allowed to go only to the government approved hospitals for giving births. They increase the demand for limited health facilities (e.g., a limited supply of beds, nurses, and doctors) in those hospitals, which then become unable to offer them to stay longer. Even nonrecipients of JSY have a low chance to stay longer there, but they can stay longer in other hospitals, which do not provide any services to JSY-recipients. In this way, we exploit the difference in hours of PHS between the treatment group (JSY recipients) and the control group (nonJSY recipients) to estimate its effect on PDCs. Applying propensity score matching (Crump et al., 2009), we ensure that this difference in the stay is not accredited to socioeconomic factors and/or quality of care that can affect stay as well as PDCs. One of the possible reasons for this difference may be the field workers who take JSY women go to the hospital and remain with them until their discharge. Field workers are often reluctant to stay longer with them because of relatively low financial incentives, and therefore, they are made to leave the hospital early (Saprii et al., 2015).

Our results show that an increase in hours of PHS reduces probabilities of complications during the first six weeks of delivery including abdominal pain, vaginal discharge, convulsion and severe headache of a mother, and fever or cold of a baby, any of which can lead to readmission. For example, the overall effect of a one day increase in the stay is a reduction of the chance of

\footnotetext{
2 In a developing country context, the main reasons for unwanted behaviors are dowry and sex of a child. If a husband's family does not receive the dowry, agreed upon at the time of marriage, from the woman's family, then that woman may undergo mental and physical abuses from her husband and his family members (Dalmia and Lawrence, 2005). This may happen if the newborn is a girl. In many developing countries, girls are not expected especially in low-income families (Palloni, 2017).
} 
abdominal pain of a mother by as high as 7 percentage points and fever or cold of a baby by 6 percentage points. If we consider heterogeneous (non-linear) effects, they are even higher: a reduction by 28 and 27 percentage points, respectively, for those who stayed less than 72 hours. The findings of negative causal effects of PHS on five out of ten PDCs is significant enough to suggest that policymakers should facilitate and encourage women and newborn for a reasonably longer fruitful stay.

After this introductory section, we briefly review the literature and state our specific contributions in Section 2 and describe data in Section 3. We establish how JSY can serve as a valid instrument in Section 34. We discuss the methodology in Section 45. We briefly describe data in Section 5 and results with robust analyses in Section 6. We write a conclusion in Section 7.

\section{Empirical Evidence}

At several points in time during the last five decades, researchers documented sharp declines in means of PHSs relating to both cesarean and vaginal deliveries. Such declines were driven by mostly cost containment, hospital bed availability and a movement toward the demedicalization of birth. For example, in Australia, the mean PHS decreased from 5.1 days in 1991 to 3.7 days in 2000 (Ford et al., 2012). In the USA, it declined steadily from 1970 until the mid-1990s (Datar and Sood, 2006; CDCP, 1995), and for example, in the cases of vaginal deliveries, the decline of it was from 2.6 days in 1992 to 1.1 days in 1995 (Ford et al., 2012). ${ }^{3}$ In the UK, 91\% of women under both cesarean and vaginal deliveries were discharged within three days of giving births in 2013-2014 as opposed to 32\% in 1975 (NHS, 2013-2014). Figure 1 shows the recent downward trends in the average days of PHSs of most of the selected OECD (the Organization for Economic Cooperation and Development) countries for which data is available for 2000, 2010 and 2015 in OECD data. There is no such data for developing countries from what we can compare PHSs over time. Campbell et al. (2016) have mentioned only a single year's PHSs (in days) for 30 low- and middle-income countries including India. From that, we can know that India has slightly lower PHS than Bangladesh but much higher PHS than Pakistan and several African nations, but we cannot tell whether the PHS has declining trends in India or other low- and middle-income countries. However, considering all kinds of deliveries in DLHS-4, the survey data we use in this study, we have found that India's average PHS declined from 96.5 hours in 2008 to 90 hours in 2013.

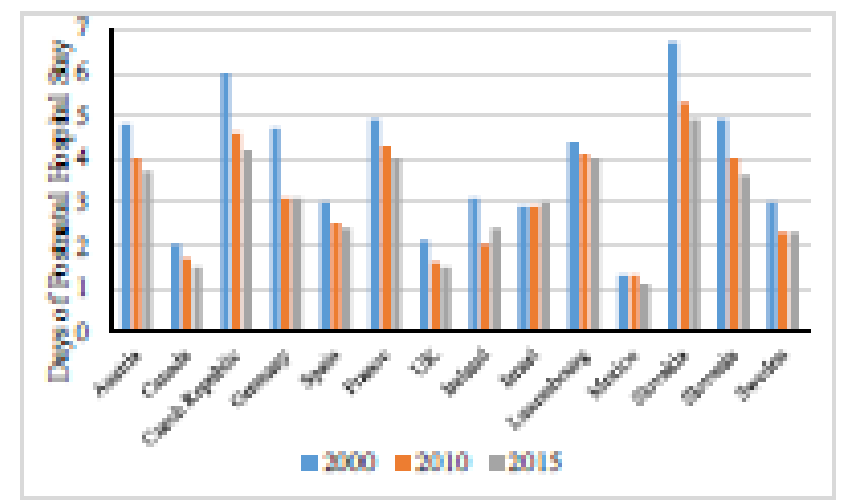

3 After introducing the minimum PHSs in the mid-1990s, the mean PHS in the USA slightly exceeded two days. 
Note: Using OECD data, we plot mean days of postnatal hospital stay of 2000, 2010, and 2015 in the bar diagram, for the selected OECD (the Organization for Economic Cooperation and Development) countries. Stays were decreasing over time in most of the countries.

Figure 1: Average days of PHS of selected OECD countries

Following sharp downward trends in the averages of PHSs, there has been a significant volume of studies estimating the effects of early discharges on readmissions or PDCs in developed countries. However, for developing countries, there exist only two studies (Gupta et al., 2006 on India and Farhat et al., 2011 on Lebanon) to the best of our knowledge. Regarding methodology used, studies can be categorized broadly into randomized controlled trials (RCTs), nonrandomized controlled trials (NRCTs), controlled before-after (CBA) studies, and interrupted time series (ITS) studies. Although RCTs are more appealing than others, a highly narrow focus either to a hospital or a small community (see Boulvain et al., 2004, Brooten et al., 1994) has made them unconvincing to generalize their results to a large population. Besides, it was always not possible to choose early and late discharge women (treatment and control groups) randomly, as women with delivery time complications tend to stay longer and vice versa (Benahmed et al., 2017). Most of the studies, including the two studies for developing countries, belong to NRCTs, which have estimated correlations or associations, as their treatment and control groups were not comparable in most of the cases. They have not considered the reverse causality or endogeneity problem, ${ }^{4}$ and have also used small samples. There are a few studies under CBA and ITS categories which have not controlled for time effects and/or endogeneity problem. In an ITS study, Evans et al. (2008) have exploited changes in U.S. federal laws in the 1990s to increase PHS. They have assumed that only PHS increased at the threshold time, other things remaining the same. Although they have addressed endogeneity problem, they have not controlled for a potential threat to time effects. There are also studies used IV regressions, but they have not tested whether instruments satisfy identification conditions. For example, Harron et al. (2017) have used the mean postnatal stay in a hospital and the number of births in a day in a hospital as instruments for PHS of an individual woman in IV regressions for the UK. While no causal relationship between stay and readmission of neonates was found for both vaginal and cesarean births, 1.4 percentage points reduction in readmission was estimated for one day increase in PHS for the subgroup of late preterm vaginal births. They have not done any validity test for their instruments, but they have reported weak predictive power of the instruments.

Results in the previous studies vary widely. Upward and downward biases might be the potential reasons. Many studies have estimated the negative correlations of PHSs with readmissions of babies and mothers (Farhat et al., 2011; Gupta et al., 2006; Johnson et al., 2002; Lee et al., 1995; Liu et al., 1997; Malkin et al., 2000a), nonurgent visits to health center and primary health care providers (Kotagal et al., 1999; Madden et al., 2002; Mandl et al., 2000), the risks of infants' jaundice (Grupp-Phelan et al., 1999; Lain et al., 2015; Liu et al., 1997), breast-feeding related problems (Gupta et al., 2006), and maternal and infant morbidities and mortalities (Danielsen et al., 2000; Lain et al., 2015; Liu et al., 1997; Malkin et al., 2000a,b; Tomashek et al., 2006). Some of those negative correlations are moderately high (in absolute term), and some are extremely high with high chances of downward biases. ${ }^{5}$ There are also many other studies, which

\footnotetext{
${ }^{4}$ For example, women with delivery time complications might have stayed longer with also higher chances of readmissions, which imply that those have higher re-admissions even stayed longer (reverse causality). In those cases, studies under NRCTs have produced upward biases (near zero estimates compared to true negative ones). There can also be downward biases when women stayed shorter have got infections, which may lead to higher chances of readmissions. In these cases, higher re-admissions have happened not only for shorter stays but also for infections.

${ }^{5}$ Using logistic regressions, Farhat et al. (2011) have found that the re-admission rate of newborn babies increased around 23 percentage points within two weeks of delivery for a one percent increase in early discharge before 48
} 
have got small or no effects of PHSs on re-admissions (Bostanci Ergen et al., 2017; Bragg et al., 1997; Brumfield et al., 1996; Cooper et al., 1996; Dalby et al., 1996; Danielsen et al., 2000; Gagnon et al., 1997; Harron et al., 2017; Johnson et al., 2002; Kotagal and Tsang, 1996; Mandl et al., 1997; Metcalfe et al., 2016), breast feeding or women's emotional well-being (Brown et al., 2004).

As the previous studies have produced highly mixed results, recent systematic reviews have found inconclusive findings (Benahmed et al., 2017; Jones et al., 2016). Indeed, there is a lack of proper causal studies. We contribute to the literature by rigorously estimating causal effects by exploiting the two-stage IV regressions. Thanks to the availability of the large survey dataset, we are able to ensure that identification conditions of an IV method are mostly satisfied by dropping observations following propensity score matching (the lowest sample we considered had 3,858 observations, see table 7). We even do joint randomization and falsification tests, which suggest that our results are robust. We have studied PHS's effects on a long list of mother's and newborn's PDCs, while some of the studies focused on a few of them. We also estimate heterogeneous effects including nonlinear effects and effects on several ethnic groups. Overall, there is no causal study in a developing country context. Our study fulfills this research gap by using an extensive survey data from one of the most prominent developing countries in the world - India.

\section{Data}

The source of our data is the District Level Household Survey (DLHS) of India, which is a repeated cross-section with different households in different waves. We use data from the fourth wave (the last one) of it (called DLHS-4), which was conducted in 2013-14 in only HPS. In this wave, household level data were collected from 300,000 households, but 73,000 women were surveyed to collect data on maternal and child health care. Women were much fewer than households, as those women who gave birth before 2008 were discarded from the survey.

About $20 \%$ of the surveyed women were the JSY beneficiaries. We drop the other programs' beneficiaries, to get a solid counterfactual group of the JSY beneficiaries. ${ }^{6}$ Individuallevel information of women is merged with their household level information, to get all required variables mentioned in the methodolgy section. To note, all data on women are self-reported by women, and household level data come from household heads. Some women did not respond to some questions (e.g., missing data problem), and thereby sample sizes are slightly different for different variables. We do not consider the previous waves of the DLHS, as they do not contain data on the key variable - PHS.

Table 1: Summary statistics

\begin{tabular}{|l|c|c|c|c|c|c|}
\hline & \multicolumn{2}{|c|}{ JSY recipients } & \multicolumn{2}{|c|}{ Nonrecipients } & & \\
\hline & \multicolumn{2}{|c|}{ (JSY=1) } & \multicolumn{2}{|c|}{ (JSY=0) } & & \\
\hline & Mean & $N$ & Mean & $N$ & Difference & p-value \\
\hline Hours of postnatal hospital stay (PHS) & 78.879 & 14,604 & 84.104 & 43,584 & -5.225 & 0.000 \\
\hline
\end{tabular}

hours in Lebanon. Such correlation has found as 16 percentage points in India (Gupta et al., 2006). Applying the same method on data from the Washington state in the USA, it has been found that a discharge before 30 hours after birth led to 28 percentage points increase in the probability of re-admission within 7 days after birth (Liu et al., 1997). In the same state, the chance of neonatal mortality increased by 26.5 percentage points because of early discharge (Malkin et al., 2000b).

${ }^{6}$ In the woman questionnaire and also in data, there are no specifications of other programmes. They are probably small-scale programs at the state or district level. 


\begin{tabular}{|c|c|c|c|c|c|c|}
\hline \multicolumn{7}{|l|}{ Post-discharge complications of mother } \\
\hline High fever $($ yes $=1$, otherwise $=0$ ) & 0.068 & 14,603 & 0.060 & 43,578 & 0.008 & 0.001 \\
\hline Abdominal pain $($ yes $=1$, otherwise $=0$ ) & 0.076 & 14,603 & 0.063 & 43,578 & 0.013 & 0.000 \\
\hline Vaginal discharge (yes $=1$, otherwise $=0$ ) & 0.029 & 14,603 & 0.022 & 43,578 & 0.007 & 0.000 \\
\hline Excessive bleeding (yes $=1$, otherwise $=0$ ) & 0.042 & 14,603 & 0.035 & 43,578 & 0.007 & 0.000 \\
\hline Convulsions (yes $=1$, otherwise $=0$ ) & 0.019 & 14,603 & 0.015 & 43,578 & 0.004 & 0.011 \\
\hline Severe headache (yes $=1$, otherwise $=0$ ) & 0.052 & 14,603 & 0.044 & 43,578 & 0.008 & 0.000 \\
\hline \multicolumn{7}{|l|}{ Post-discharge complications of newborn } \\
\hline Breast feeding or drinking poorly (yes $=1$, otherwise $=0$ ) & 0.051 & 14,603 & 0.048 & 43,583 & 0.003 & 0.251 \\
\hline Fever or cold to touch (yes $=1$, otherwise $=0$ ) & 0.036 & 14,603 & 0.033 & 43,583 & 0.003 & 0.141 \\
\hline Fast or difficult breathing (yes $=1$, otherwise $=0$ ) & 0.028 & 14,603 & 0.024 & 43,583 & 0.004 & 0.015 \\
\hline Blood in stool (yes $=1$, otherwise $=0$ ) & 0.007 & 14,603 & 0.006 & 43,583 & 0.001 & 0.478 \\
\hline \multicolumn{7}{|l|}{ Socioeconomic characteristics } \\
\hline Wealth index & -0.579 & 14,599 & 0.440 & 43,556 & -1.019 & 0.000 \\
\hline Mother's age at birth (in year) & 23.885 & 14,604 & 24.810 & 43,584 & -0.925 & 0.000 \\
\hline Years of education completed by mother & 8.769 & 12,748 & 10.099 & 38,902 & -1.330 & 0.000 \\
\hline Years of education completed by husband & 8.888 & 13,068 & 10.321 & 40,154 & -1.433 & 0.000 \\
\hline Hindu (yes $=1$, otherwise $=0$ ) & 0.706 & 14,602 & 0.714 & 43,570 & -0.008 & 0.048 \\
\hline Scheduled caste $($ yes $=1$, otherwise $=0$ ) & 0.310 & 13,980 & 0.227 & 40,966 & 0.083 & 0.000 \\
\hline Tribe $($ yes $=1$, otherwise $=0$ ) & 0.173 & 14,597 & 0.098 & 43,508 & 0.075 & 0.000 \\
\hline Poor (yes $=1$, otherwise $=0$ ) & 0.468 & 14,601 & 0.310 & 43,567 & 0.158 & 0.000 \\
\hline Birth order & 1.822 & 14,553 & 1.950 & 43,252 & -0.128 & 0.000 \\
\hline Rural (yes=1, otherwise $=0$ ) & 0.669 & 14,604 & 0.528 & 43,584 & 0.141 & 0.000 \\
\hline \multicolumn{7}{|l|}{ Delivery time complications of mother } \\
\hline Premature labour (yes $=1$, otherwise $=0$ ) & 0.112 & 14,602 & 0.117 & 43,579 & -0.005 & 0.120 \\
\hline Excessive bleeding (yes $=1$, otherwise $=0$ ) & 0.057 & 14,602 & 0.051 & 43,579 & 0.006 & 0.004 \\
\hline Prolonged labour $($ yes $=1$, otherwise $=0$ ) & 0.092 & 14,602 & 0.075 & 43,579 & 0.017 & 0.000 \\
\hline Obstructed labour (yes $=1$, otherwise $=0$ ) & 0.098 & 14,602 & 0.094 & 43,579 & 0.004 & 0.231 \\
\hline Breech presentation (yes $=1$, otherwise $=0$ ) & 0.022 & 14,602 & 0.024 & 43,579 & -0.002 & 0.182 \\
\hline Convulsions (yes $=1$, otherwise $=0$ ) & 0.031 & 14,602 & 0.034 & 43,579 & -0.003 & 0.072 \\
\hline cesarean $($ yes $=1$, otherwise $=0$ ) & 0.194 & 14,604 & 0.272 & 43,584 & -0.078 & 0.000 \\
\hline \multicolumn{7}{|l|}{ Health facility types } \\
\hline Sub-health center $($ yes $=1$, otherwise $=0)$ & 0.027 & 12,004 & 0.017 & 32,509 & 0.010 & 0.000 \\
\hline Primary health center $($ yes $=1$, otherwise $=0$ ) & 0.205 & 12,004 & 0.098 & 32,509 & 0.107 & 0.000 \\
\hline Community health center (yes $=1$, otherwise $=0$ ) & 0.092 & 12,004 & 0.050 & 32,509 & 0.042 & 0.000 \\
\hline Sub-district or district hospital (yes $=1$, otherwise $=0$ ) & 0.549 & 12,004 & 0.376 & 32,509 & 0.173 & 0.000 \\
\hline
\end{tabular}

Note: Using primary samples (excluding home deliveries and PHSs above 500 hours) from the household and woman surveys of DLHS-4, we show summary statistics (observations and means by JSY and nonJSY women, mean differences and p-values) of all relevant variables used in this study, excluding state and birth year dummies. This is a vital table, as we can understand whether our instrument is valid. As most of the control variables (socioeconomic variables, delivery time complications and proportions of deliveries in health facility types) have statistically and significantly different means between JSY and nonJSY women, the validity of the instrument is questioned in the primary samples.

In Table 1, we shows means and numbers of observations (e.g., numbers of mothers) of variables (excluding state dummies and birth year dummies) used in this study, by treatment and control groups of JSY. Differences in means of those variables between that two groups and pvalues of such differences are also shown there. In the variable list, hours of PHS is our $X_{i}$, and each PDC dummy is $Y_{i}$. Other variables under socioeconomic conditions, delivery time complications, and health facility types are control variables. All of these control variables plus state and birth year dummies might have effects on both $X_{i}$ and $Y_{i}$. Control variables are, in general, deciders of how long a mother stays in a hospital after the delivery and how much care she receives at home after the discharge and thus how much probabilities of PDCs she may have. For example, a mother from a wealthy household may stay longer and may have better care at 
home and lower probabilities of PDCs. Delivery time complications evidently lead to longer stays and higher chances of PDCs. Government health facilities have poorer facilities than private ones, and mothers may stay shorter there and may have higher chances of PDCs.

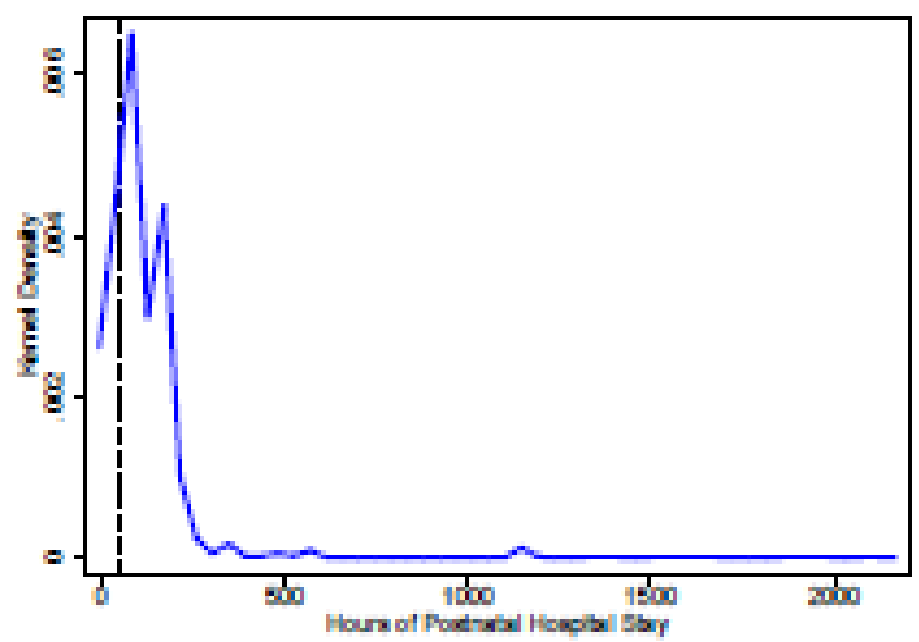

Note: Using woman survey of DLHS-4, we plot the density of hours of postnatal hospital stay, for those women who took institutional delivery services, with the epanechnikov kernel and bandwidth 7.05. To see how the density changes we draw the dashed vertical line at 48 hours used as a threshold for early discharge by most of the studies; however, the mean stay is 92.58 hours. We discard observations for stays above 500 hours, and in this way, we lose 911 outliers. Our primary samples exclude home deliveries and these outliers. Table (1) also shows summary statistics with the primary samples.

Figure 2: Kernel density plot of hours of postnatal hospital stay in the cases of institutional deliveries

Except for hours of PHS and wealth index, Aall variables are generated in a straight way except hours of PHS and wealth index, implying that we have retained the original values without deriving new forms of these variables. In the survey data, a length of PHS was measured in hours if a woman's stay was less than or equal to 48 hours, and otherwise, it was measured in days. We convert days into hours by multiplying the number of days with 24 hours, to have hours as a common measure of the unit. In this way, we incur the measurement error in $X_{i}$, as all mothers may not have stayed full hours of the last day. For example, if a mother said that she stayed three days, which does not necessarily mean that she stayed 72 hours. She might have been released between 48 and 72 hours. In this case, estimation of hours will be wrong. However, we cannot get rid of the measurement error in $X_{i}$. If we constructed $X_{i}$ in the opposite way (e.g., converting hours into days), there would have also been the measurement error in days of PHS. ${ }^{7}$ The measurement error is another source of the endogeneity of $X_{i}$.

In Figure (2), we plot the kernel density function of $X_{i}$, where mothers/observations are mostly distributed below roughly 500 hours or approximately 21 days. Above that, there are some outliers. The dashed vertical line is a reference line at 48 hours used as a threshold for the early discharge by most of the previous studies, but most of the mothers stayed around four days in hospitals after deliveries (e.g., the mean stay is 92.58 hours). As outliers may bias the true causal effects, we drop 911 observations in which PHSs are above 500 hours. We get our primary samples excluding these outliers and births at home.

Wealth index is constructed by applying principal component analysis over a list of wealth

\footnotetext{
7 However, we divide $X_{i}$ and the predicted value of it by 24 in equations (1) and (4), to scale up the values of $\rho$.
} 
of a household - cooking fuel, house type, number of dwelling rooms, electricity, house ownership, landholding, radio, television, computer, internet, telephone, mobile phone, washing machine, refrigerator, sewing machine, watch, bicycle, motorcycle, car, tractor, tube well, cart, air cooler.

\section{The Instrumental Variable}

As argued above, the estimated effect of PHS on PDCs using simple logistic regressions can be biased upwards or downwards respectively due to the low quality of hospital care or some socioeconomic characteristics (e.g., dowry, sex of the baby) influencing both PHS and PDCs. To correct for this endogeneity, we run instrumental variables regressions. We need an instrument that will exogenously cause PHS to vary, controlling for socioeconomic characteristics including both observables and unobservables. We exploit a national cash transfer program Janani Suraksha Yojana (JSY) in India to define the treatment and the control groups. Below we justify why JSY dummy is supposed to cause PHS to vary exogenously between the two groups. In doing so, we first justify why JSY may lead to lower PHS specifically for the treatment group. Then we establish that the variation is exogenous by controlling for all possible observable characteristics and thus the instrument satisfies identification conditions.

\subsection{Why Is Janani Suraksha Yojana (JSY) an Instrument?}

In April 2005, the prime minister of India launched JSY under the National Rural Health Mission (NRHM), with the ultimate goal of reducing maternal and neonatal mortality. It was evident that increasing institutional delivery can achieve such a goal, especially among poorer pregnant women. Therefore, the primary objective of this program is to increase the number of institutional deliveries. In addition to this, it also works to increase the uptake of antenatal and postnatal care services.

JSY is one of the most extensive cash transfer programs in the world, covering more than 10 million women per year (registrar2013special). It is a universal program in 10 low performing states (LPS) - Uttar Pradesh, Uttaranchal, Bihar, Jharkhand, Madhya Pradesh, Chhattisgarh, Assam, Rajasthan, Orissa, and Jammu and Kashmir - where rates of institutional delivery were meager. ${ }^{8}$ In these states, all pregnant women delivering in public or accredited private institutions are eligible. The program is means tested in the rest of the states, called high performing states (HPS) where those women qualify for the program, whose households have a below poverty line card or scheduled caste status or tribal status, and whose ages are 19 years and above, and who give births in public or accredited private institutions, and whose birth orders are maximum two.

In both LPS and HPS, the government has employed field workers, called accredited social health activists (ASHAs), to work as intermediaries between JSY administrators and pregnant women. They identify the eligible pregnant women, and functional government health centers and accredited private health institutions, for them. Both the eligible pregnant woman and an ASHA receive cash incentives. In LPS (HPS), they receive 1,400 (700) and 600 (600) Rupees respectively in the rural area but 1,000 (600) and 400 (400) in the urban area. They usually receive their entitled money in one go at the health center, immediately on arrival. On the same visit, the woman is registered for the institutional delivery (with the entitlement of postnatal care), and for three antenatal care services before delivery. In the case of an emergency, any obstetric care such as

\footnotetext{
8 If institutional delivery is less than $25 \%$ in a state, that is called low-performing state; otherwise, that is called high-forming state.
} 
cesarean section is also provided with additional financial support from JSY [12].

A JSY beneficiary has to consider a high amount of out-of-pocket (OOP) expenditure for an institutional delivery, and that OOP is extremely higher than the JSY cash payment (Modugu2012). In spite of that, studies found that JSY significantly increases the number of institutional deliveries (see powell2015financial; Carvalho2014; Gopalan2012; Gupta2011; Gupta2012; Lim2010; Modugu2012).

To claim that the JSY dummy is a valid instrument, we establish our hypothesis: the JSY recipients stay shorter after deliveries in the hospitals than the control women. For that, we use woman and facility surveys of DLHS-4. We first try to prove that JSY increases the demand for institutional delivery services in those hospitals which provide that services to the JSY women. Then we show that given the limited supply of beds, staff, machines, medicines and other infrastructure, an increase in demand creates an excess demand for institutional deliveries, which leads the JSY women to stay shorter in those JSY service providing hospitals compared to the nonrecipients of JSY who are free to go to any hospital.

In Table (2), the woman survey says that more than $80 \%$ of the JSY women utilized institutional delivery services from public health facilities - Sub-health center (SHC), primary health center (PHC), community health center (CHC), sub-divisional hospital (SDH) and district hospital (DH) (see Appendix A for their explanations). In contrast, without the program, most women delivered at private hospitals or home. These findings imply that JSY increases the demand for institutional delivery services in the public health system. We shall get a clearer picture from the facility survey.

The facility survey collected data on public health facilities from both high and low performing states, but we use data from only HPS as the woman survey was conducted in just HPS. In Table (3), we show how many government health facilities provide institutional delivery services to which proportions of the JSY women. It should be noted that surveyors collected data on the number of JSY recipients received institutional delivery services from a facility, from the record book of that facility. We see that only less than 5\% of the SHCs do not provide any institutional delivery services to the JSY women, but in around 91\% of the SHCs, the proportion of the JSY women taken that services are 0.75 or above. These imply that the SHCs provide that services mainly to the JSY women. But from Appendix A, we know that the SHCs do not have any bedding facilities and any qualified doctors, which indicate that they have limited capacity to offer the JSY women to stay longer. An almost similar picture is seen in other public health facilities. The proportion of JSY women is 0.75 or above in $59.29 \%$ of the PHCs, $54.11 \%$ of the CHCs, and $48.64 \%$ of the SDHs and the DHs.

Table 2: Different numbers of JSY and nonJSY women delivered their last babies in different

places
\begin{tabular}{|l|c|c|c|c|c|c|}
\hline & \multicolumn{2}{l}{ JSY recipients (JSY=1) } & $\begin{array}{c}\text { Nonrecipients } \\
\text { (JSY=0) }\end{array}$ & \multicolumn{3}{c|}{ Total } \\
\hline Place of Last Delivery & $N$ & $\%$ & $N$ & $\%$ & $N$ & $\%$ \\
\hline Sub-health Center (SHC) & 327 & 2.48 & 563 & 1.22 & 890 & 1.5 \\
\hline Primary Health Center (PHC) & 2,492 & 18.88 & 3,274 & 7.12 & 5,766 & 9.74 \\
\hline Community Health Center (CHC) & 1,114 & 8.44 & 1,632 & 3.55 & 2,746 & 4.64 \\
\hline District Hospital (DH)/Sub-district Hospital (SDH) & 6,696 & 50.73 & 12,446 & 27.07 & 19,142 & 32.34 \\
\hline United Health Care (UHC) & 294 & 2.23 & 508 & 1.1 & 802 & 1.36 \\
\hline Dispensary/Clinic & 66 & 0.5 & 127 & 0.28 & 193 & 0.33 \\
\hline AYUSH Hospital/Clinic & 5 & 0.04 & 7 & 0.02 & 12 & 0.02 \\
\hline
\end{tabular}




\begin{tabular}{|l|c|c|c|c|c|c|}
\hline Private Dispensary/Clinic & 74 & 0.56 & 730 & 1.59 & 804 & 1.36 \\
\hline Private Hospital & 1,074 & 8.14 & 13,608 & 29.59 & 14,682 & 24.81 \\
\hline Private AUSH Hospital/Clinic & 4 & 0.03 & 29 & 0.06 & 33 & 0.06 \\
\hline nongovernment Organisation (NGO) & 17 & 0.13 & 73 & 0.16 & 90 & 0.15 \\
\hline On the Way of Hospital & 14 & 0.11 & 48 & 0.1 & 62 & 0.1 \\
\hline At Home & 925 & 7.01 & 12,008 & 26.11 & 12,933 & 21.85 \\
\hline At Parents Home & 66 & 0.5 & 532 & 1.16 & 598 & 1.01 \\
\hline Work Place & 1 & 0.01 & 30 & 0.07 & 31 & 0.05 \\
\hline Other & 30 & 0.23 & 367 & 0.8 & 397 & 0.67 \\
\hline Total & 13,199 & 100 & 45,982 & 100 & 59,181 & 100 \\
\hline
\end{tabular}

Note: Using the woman survey of DLHS-4, we show the number and percentage of women delivered their last children, by the places of deliveries and JSY status. We see that most of the JSY women delivered their last children in public health facilities, which imply that they increased the demand for institutional delivery services in the public health facilities.

Table 3: Number of health facilities provided services to JSY women

\begin{tabular}{|l|c|c|c|c|c|c|c|c|}
\hline & \multicolumn{2}{|c|}{ SHC } & \multicolumn{2}{c|}{ PHC } & \multicolumn{2}{c|}{ CHC } & \multicolumn{2}{c|}{ DH/SDH } \\
\hline Proportion of JSY women & $N$ & $\%$ & $N$ & $\%$ & $N$ & $\%$ & $N$ & $\%$ \\
\hline 0 & 344 & 4.88 & 608 & 15 & 183 & 9.01 & 80 & 8.06 \\
\hline-0.25 & 103 & 1.46 & 267 & 6.59 & 190 & 9.35 & 144 & 14.5 \\
\hline $.25-0.50$ & 131 & 1.86 & 419 & 10.34 & 256 & 12.6 & 139 & 14 \\
\hline $.50-0.75$ & 73 & 1.04 & 356 & 8.78 & 303 & 14.92 & 147 & 14.8 \\
\hline $.75-1$ & 6,394 & 90.76 & 2,403 & 59.29 & 1,099 & 54.11 & 483 & 48.64 \\
\hline Total & 7,045 & 100 & 4,053 & 100 & 2,031 & 100 & 993 & 100 \\
\hline
\end{tabular}

Note: The facility survey of DLHS-4 collected data on government health facilities. Using that survey, by the government health facility type, we report how many government health facilities provide institutional delivery services to which proportions of JSY recipients among all women received that services. We see that more than $90 \%$ of sub-health centers, $59.29 \%$ of primary health centers, $54.11 \%$ of community health centers and $48.64 \%$ of district/sub-district hospitals provide institutional delivery services to the women, of whom $75 \%$ or above are JSY recipients. These are another evidence that JSY increases the demand for that services.

The overall picture again says that the JSY women utilize the public health facilities in higher proportion compared to those women who did not receive JSY. The main reason is that the government assigns mainly the public health facilities to provide services to the JSY women. Accredited private health facilities that provide health services to the JSY women are few, as they are concentrated mainly in the urban area. Besides, data from the woman survey says that there are lower out-of-pocket expenditures (self-reported by women) in the public health facilities than that of private institutions.

Now, we check the supply/service readiness of the public health facilities that is whether they can meet the extra demand for the institutional delivery services caused by the program. To do that, we run simple bi-variate OLS regressions of several supply-side (service readiness) variables on the number of the JSY women taken the institutional delivery service (see Table (4)). It should be noted that all supply-side variables are not available for each facility and the surveyors collected their data from the visual inspections or record books of the facilities. In the Table, we first regress the proportion of institutional deliveries (total number of institutional deliveries divided by total number of services provided by the health institute in a year) on the independent variable. We find a positive result in each case but statistically significant in the case of only PHC. Negative results under sub-health center imply that the SHCs that provide services to the JSY women have lower chances of trained nurses and proper labor rooms (with mackintosh sheet, suction machine, sterilizer, 24 hours water supply and inverter). In other words, the JSY program is run in the poor areas where the SHCs also have poor provisions of health facilities. In contrast, 
the indicators of a proper labor room have positive results in the cases of the primary health center and community health center, which imply that chances of appropriate labor rooms in the PHCs and the CHCs increase with the increase in the number of institutional delivery services taken by the JSY women. However, the most important point is that the availability of several kinds of staff per delivery decreases with the increase in the number of JSY women in PHCs, CHCs, and SDHs and DHs (see negative results of staff, such as, surgeon, physician, nurse etc. under PHC, CHC and DH/SDH). Bedding and cleanliness of labor room data are available in only SDHs and DHs, and they show negative results.

We can derive several points from the above results. First, JSY exogenously increases the demand for institutional delivery services in the public health system, which is not ready to meet the extra demand. Second, this demand-supply gap may force the JSY women to stay shorter in the hospital after deliveries. Third, PHSs of the control women may also be lower in those public health facilities that provide services to the JSY women, but that will likely be higher in other health facilities. We cannot merge the woman survey data with the facility data. Otherwise, we could have known a woman went to which facility, and in this way, we could have identified stay time by the specific facility in a locality. However, the above results are substantial to establish our hypothesis that JSY women stay shorter than nonrecipients of JSY. Another important point should be noted that there might be a higher chance of infection for JSY women than their counterparts, as we have seen in Table (4) that cleanliness decreases with the increase in the number of JSY women taken institutional delivery services from the government hospital. We discuss in the following subsection how the chance of infection can influence our causal estimates.

Table 4: Effects of Janani Suraksha Yojana on service readiness of the government health facilities

\begin{tabular}{|c|c|c|c|c|c|c|c|c|c|c|c|c|}
\hline & \multicolumn{3}{|c|}{ SHC } & \multicolumn{3}{|c|}{ PHC } & \multicolumn{3}{|c|}{$\mathrm{CHC}$} & \multicolumn{3}{|c|}{$\mathrm{SDH} / \mathrm{DH}$} \\
\hline Service Readiness (Dependent Variables) & Coeff. & t-ratio & $N$ & Coeff. & t-ratio & $N$ & Coeff. & t-ratio & $N$ & Coeff. & t-ratio & $N$ \\
\hline Proportion of institutional deliveries & 0.041 & 1.56 & 7,032 & $0.0003 * * *$ & 3.64 & 4,044 & 0.0006 & 1.17 & 2,006 & 0.0237 & 0.89 & 976 \\
\hline Female skilled birth attendant was trained & $-0.001 * * *$ & -6.68 & 3,321 & 0.0010 & 1.50 & 1,345 & $0.0008 * *$ & 2.35 & 1,024 & 0.0001 & 1.44 & 386 \\
\hline Mackintosh sheet in labor room & $-0.001 * * *$ & -4.57 & 2,099 & $0.0013 * * *$ & 3.48 & 3,642 & $0.0004 * * *$ & 2.66 & 2,016 & & & \\
\hline Suction machine in labor room & $-0.002 * * *$ & -7.41 & 2,099 & $0.0011^{* *}$ & 2.31 & 3,594 & $0.0006^{* * *}$ & 3.98 & 2,017 & & & \\
\hline Sterilizer in labor room & $-0.003 * * *$ & -8.96 & 2,098 & $0.0014 * * *$ & 3.14 & 3,597 & $0.0005 * * *$ & 3.76 & 2,017 & & & \\
\hline hours water supply in labor room & $-0.001 * * *$ & -3.14 & 2,099 & $0.0015^{* * *}$ & 3.25 & 3,593 & $0.0004 * *$ & 1.98 & 2,017 & & & \\
\hline Inverter connected in labor room & $-0.002 * * *$ & -9.79 & 2,093 & & & & & & & & & \\
\hline Clean labor room & & & & & & & & & & -0.0001 & -1.17 & 991 \\
\hline Surgeon per institutional delivery & & & & & & & $-0.0004 * * *$ & -3.93 & 1,849 & 0.0004 & 0.38 & 956 \\
\hline Physician per institutional delivery & & & & & & & $-0.0003 * *$ & -2.11 & 1,851 & 0.0004 & 0.37 & 956 \\
\hline Gynecologist per institutional delivery & & & & & & & $-0.0003 * *$ & -2.20 & 1,851 & 0.0004 & 0.31 & 955 \\
\hline Medical officer per institutional delivery & & & & $-0.0040 * * *$ & -7.32 & 2,982 & & & & & & \\
\hline Nurse per institutional delivery & & & & $-0.0037 * * *$ & -6.89 & 2,982 & $-0.0024 * * *$ & -5.65 & 1,851 & $-0.0009 * * *$ & -5.04 & 956 \\
\hline Pharmacist per institutional delivery & & & & $-0.0033 * * *$ & -7.42 & 2,981 & $-0.0008 * * *$ & -6.17 & 1,851 & $-0.0003 * * *$ & -3.06 & 956 \\
\hline Field worker per institutional delivery & & & & $-0.0071 * * *$ & -7.21 & 2,982 & & & & & & \\
\hline Bed per institutional delivery & & & & & & & & & & $-0.0042 * * *$ & -4.90 & 955 \\
\hline
\end{tabular}

Note: Using simple OLS method and the facility survey of DLHS-4, we run bi-variate regressions of all above service readiness variables on only the number of JSY recipients taken institutional delivery service from the government health facility. We generate service readiness variables in a straight way. The first variable is the number of institutional deliveries divided by the total number of patients served by the health facility. From the second to the seventh variable in the above list, they are just dummy variables with 1 if yes and 0 if no. The rest of the variables are respective staff and beds divided by the total number of institutional delivery services provided by the health institute. For each regression, along with the coefficient, we also report t-value estimated from robust standard error, and the number of health facilities, $N . * p<0.10,{ }^{*} p<0.05, * * * p<0.01$.

\subsection{Identification}

In the case of an IV method we use, there are two identification conditions needed to be satisfied to get an unbiased estimate of the causal effect. First, the instrument must be powerful. 
In our case, our instrument, JSY dummy ( 1 if a woman received financial incentives from JSY, 0 if she did not receive any financial incentives) must be a powerful predictor of PHS. As we mention in the results section, JSY dummy is a powerful instrument, as it easily satisfies the criterion for the power of $F=10$ in the first stage (stock2002survey). Second, the instrument must be valid. This means that the instrument must be uncorrelated with the error term in the second stage. In our case, JSY dummy must be uncorrelated with the woman's residual probability of a PDC after controlling for the predicted PHS and observed socioeconomic characteristics of the woman.

It is always difficult to prove the validity of an instrument because some doubt will always remain. However, the validity of JSY dummy would be questioned if observed socioeconomic characteristics, that are correlated with PDCs, are not randomly distributed among JSY beneficiaries and nonbeneficiaries of JSY. One may argue that PDCs are different between these two groups because socioeconomic characteristics are different and not because PHSs are different. As the JSY program provides cash incentives to the disadvantaged women, observed socioeconomic characteristics are likely to be different between the two groups of women (e.g., the JSY beneficiaries are likely to be poorer than the control women).

Our primary samples show that means of most of the observed socioeconomic characteristics are statistically and significantly different between the treatment and the control groups (see Table 1). These imply that our instrument, JSY dummy, is not valid in the primary sample. To increase the validity, we dropped some observations based on the propensity score of JSY estimated after running a logit regression of JSY dummy on observed socioeconomic characteristics and other control variables. To hold the overlapping (or common support) condition, such dropping is suggested by [3], in the cases of matching estimators. We further restrict samples to those births, which are not only the first births of the women but also the first births in their households in a period. ${ }^{9}$ After dropping some observations in these ways, differences in means of most of the observed socioeconomic characteristics (between the JSY women and the control women) become statistically insignificant (and also the propensity score of JSY overlaps between JSY and nonJSY women), but the difference in the mean PHS becomes statistically and significantly different and larger (in the absolute value) than before, and differences in means of the half of available PDCs become statistically significant (see Table (7), Sample3). So, the raw estimates from the final subsamples (Sample3) tell us that with the increase in the validity of the instrument, PHS has negative effects on half of the available PDCs.

Delivery time complications (e.g., premature labor, excessive bleeding, etc.) can also increase the chances of PDCs even though PHS was sufficient. If the treatment group of JSY has a higher chance of delivery time complications than the control group, the negative causal estimates will be inflated. In our final subsample (Sample3), the chances of delivery time complications are balanced between the treatment and the control groups of JSY. So, we can rule out any biases caused by them. Another important source of biases can be the infection, which is a critical factor in a developing country context. In general, the infection can make negative causal estimates very low (in absolute terms)or zero (i.e., upward biases) because a longer stay leads to a higher chance of infection and then PDCs. In our setting, the infection can make our IV estimates biased, but differently. As we have seen in Table (4) that JSY women have a higher chance of infection than nonJSY women (e.g., cleanliness decreases with the increase in the number of JSY recipients in the health facility), the former group will have a higher chance of PDCs in addition to the fact that they stayed shorter than the latter group. In this way, the negative causal estimates

\footnotetext{
9 That means we initially drop those households, which have more than one woman having births, and then we drop those births, which are not first of the women.
} 
will exceed the true causal estimates (in absolute terms). In the other way, if the control group of JSY also go to the same hospitals where the treatment group of JSY went, the chances of infections will be balanced between two groups, and biases caused by infections can be removed. But if the control group of JSY has a higher chance of infection because of their longer stay than the treatment group of JSY, then biases due to infections can be typical (e.g., upward biases). As we mentioned in the previous subsection, we cannot merge the woman survey with the facility survey. We cannot know whether JSY recipients and nonrecipients went to the same health facilities in same proportions. However, we can know how much proportions of JSY and nonJSY women went to the specific type of health facility. As most of the JSY recipients went to the public health facilities, we need to know whether proportions of two groups of women are same in each public health facility type. In our final subsample, they are balanced in each public health facility type (see Table 7, Sample 3). Thus, we can claim that infection becomes less influential in making our causal estimates biased.

\section{Methodology}

$$
\begin{aligned}
& \text { Our structural model is, } \\
& \qquad Y_{i}=\alpha+\rho X_{i} / 24+\beta_{1} Z_{1 i}+\beta_{2} Z_{2 i}+\cdots+\beta_{k} Z_{k i}+\varepsilon_{i},
\end{aligned}
$$

where $Y_{i}$ is a dummy variable for a PDC of mother or child $i$ (1 if had a complication, 0 not), $\alpha$ is the constant term, $\rho$ is the coefficient of $X_{i} / 24$ where $X_{i}$ is hours of PHS of mother or child $i$, and this $\rho$ is the parameter of interest. The value of $\rho$ will tell us how much the probability of a PDC is changed with one day increase in PHS. Based on the literature, we expect that the value of $\rho$ will be negative, as a shorter PHS may lead to a higher chance of a PDC, and vice versa.

In the above structural model, we also include $k$ number of control variables, $Z_{1 i}, Z_{2 i}, \ldots, Z_{k i}$, and $\beta_{1}, \beta_{2}, \ldots, \beta_{k}$ are their respective coefficients. Control variables include socioeconomic conditions and delivery time complications of mother $i$, and dummies for four types of main government hospitals (see Appendix A for their descriptions) where mothers delivered their first babies. These control variables can influence the PDCs. To control for state heterogeneity and time effects, state dummies and dummies for delivery years are also included in the list of the control variables.

However, equation (1) is not our desired model, as it can give us a biased result of $\rho$, as $X_{i}$ is likely to be an endogenous variable such as $E\left(X_{i} \varepsilon_{i}\right) \neq 0$. We think that there are some unobserved factors such as dowry, which are included in $\varepsilon_{i}$, can affect both PHS and PDCs. To remove the endogeneity of $X_{i}$, we have to consider instrumental variables (IV) regression models to get an unbiased result of $\rho$.

The first stage regression model (the reduced form equation for $X_{i}$ ) of IV regression models is as follows,

$$
X_{i}=\delta+\gamma J S Y_{i}+\theta_{1} Z_{1 i}+\theta_{2} Z_{2 i}+\cdots+\theta_{k} Z_{k i}+\eta_{i}
$$

where we see that hours of PHS, $X_{i}$, depends on $J S Y_{i}$ dummy and $k$ number of control variables, $Z_{1 i}, Z_{2 i}, \ldots, Z_{k i}$. It should be noted that all control variables used in (1) should also be used in (2), as all of them have direct effects on hours of PHS. Here parameters and the error term have different notations, as the dependent variable is now $X_{i}$.

As the instrument should not have any direct effect on $Y_{i}$, we cannot have a straight model for $Y_{i}$ with $J S Y_{i}$. However, we can get such model indirectly. We can derive reduced form equation for $Y_{i}$ through the reduced form equation for $X_{i}$ (equation (2)), if we substitute equation (2) into equation (1). So, after that substitution, the reduced form equation for $Y_{i}$ is as follows, 


$$
Y_{i}=\kappa+\gamma \rho J S Y_{i}+\lambda_{1} Z_{1 i}+\lambda_{2} Z_{2 i}+\cdots+\lambda_{k} Z_{k i}+\zeta_{i},
$$

where $\kappa=\alpha+\delta \rho, \lambda_{1}=\theta_{1} \rho+\beta_{1}, \lambda_{2}=\theta_{2} \rho+\beta_{2}, \lambda_{k}=\theta_{k} \rho+\beta_{k}$, and $\zeta_{i}=\rho \eta_{i}+\varepsilon_{i}$. We can now estimate $\rho$ through indirect least squares (ILS), which will give us an unbiased value of it. In the case of ILS, if we run two separate regressions of equations (2) and (3), and then we divide the coefficient of $J S Y_{i}$ in (3) by the coefficient of $J S Y_{i}$ in (2), we will get the value of $\rho$ easily. However, ILS method does not give us standard error and other diagnostic results. Rather, we can run two stage least squares (2SLS), or IV, or generalized method of moment (GMM) regression, as all of them will give us standard error and diagnostic results automatically in addition to the unbiased value of $\rho$. For $2 \mathrm{SLS}$, or IV, or GMM regression, we can now consider the following equation for $Y_{i}$.

$$
Y_{i}=\alpha+\rho E\left(X_{i} \mid J S Y_{i}\right) / 24+\beta_{1} Z_{1 i}+\beta_{2} Z_{2 i}+\cdots+\beta_{k} Z_{k i}+\varepsilon_{i},
$$

which is slightly different from equation (1). Now, the actual $X_{i}$ is replaced by the predicted/estimated $X_{i}, E\left(X_{i} \mid J S Y_{i}\right)$. If we want to run 2SLS regression, we have to run a regression of equation (2) first, and then generate predicted value of $X_{i}$ from this regression, and then run a regression of equation (4) with that predicted value of $X_{i}$. We could also run IV and GMM regressions of (4), but they have slightly different ways of estimating parameters (see wooldridge2010econometric, davidson2004econometric). They do not consider logit or probit model for a dummy dependent variable, which is much more required if the average value of dummy dependent variable is very low or near zero. Otherwise, they may produce a biased standard error of $\rho$.

In DLHS-4, proportions/means of PDCs are found very low (below 0.10 or 10\%), and we need to consider logit or probit model for $Y_{i}$. As logit model is widely used, we choose it to run regressions of equations (1) and (4), but the predicted value of $X_{i}$ in (4) comes from an OLS regression of equation (2). It should be noted that the estimate of $\rho$ in (1) will give us the association while that in (4) will give the causal effect. Although we desire to estimate causal effects, we also estimate associations for comparisons.

\section{Results}

\subsection{Results with primary samples}

\subsubsection{Summary statistics and the validity of the instrument}

In Table 1 in data section (section 3), p-values indicate that means of almost all variables are statistically and significantly different between treatment and control groups of JSY. We expect statistically significant differences in means of only hours of PHS, $X_{i}$, and each PDC dummy, $Y_{i}$. Statistically significant differences in means of the control variables imply the violation of the second identification condition of an IV method. This means that the control variables are not randomly distributed between that two groups. Now, one can argue that differences in means of PDCs are due to differences in means of the control variables not the difference in means of $X_{i}$. However, the JSY dummy is found as a powerfulgood predictor of hours of PHS, as the F-value is 12.125 , which satisfies the criterion for the power of $\mathrm{F}=10$ in the first stage (stock2002survey). So, the first identification condition of an IV method is satisfied here. However, the instrument is not valid, as the second identification condition is not satisfied. We shall address this issue in the 
next subsection.

\subsubsection{Regression results}

For each PDC dummy, $Y_{i}$, we first run logit regressions of the structural equation (1). In the nonIV columns of Table (5), we show marginal effects of $X_{i} / 24$ on the probabilities of PDCs with their p-values (estimated from robust standard errors) in parentheses. We see that they are near zero, but statistically significant in all cases. Another interesting matter is that we expect negative signs of them, but they are positive here. This is more likely to be due to the low-quality services of the hospitals which are common in a developing country like India, that may cause increased complications with longer stay (and also may be due to infections). Thus, we consider that the structural model gives us biased results because of the endogeneity of $X_{i}$, and we cannot rely on these results.

Table 5: Estimates of $\hat{\rho}$ from running regressions of equations (1) and (4) which are nonIV/structural and IV equations

\begin{tabular}{|l|c|c|c|c|c|c|}
\hline & \multicolumn{3}{|c|}{ NonIV } & \multicolumn{3}{c|}{ IV } \\
\hline & Estimate & p-value & $N$ & Estimate & p-value & $N$ \\
\hline Post-discharge complications of mother & & & & & & \\
\hline High fever (yes=1, otherwise=0) & $0.002^{* * *}$ & $(0.000)$ & 36,691 & -0.030 & $(0.181)$ & 34,599 \\
\hline Abdominal pain (yes=1, otherwise=0) & $0.003^{* * *}$ & $(0.000)$ & 36,691 & $-0.062^{* * *}$ & $(0.004)$ & 34,599 \\
\hline Vaginal discharge (yes=1, otherwise=0) & $0.000^{*}$ & $(0.079)$ & 36,691 & $-0.024^{* *}$ & $(0.043)$ & 34,599 \\
\hline Excessive bleeding (yes=1, otherwise=0) & $0.001^{* * *}$ & $(0.000)$ & 36,691 & $-0.028^{*}$ & $(0.074)$ & 34,599 \\
\hline Convulsion (yes=1, otherwise=0) & $0.000^{* *}$ & $(0.014)$ & 36,588 & $-0.021^{* *}$ & $(0.036)$ & 34,502 \\
\hline Severe headache (yes=1, otherwise=0) & $0.002^{* * *}$ & $(0.000)$ & 36,691 & $-0.052^{* * *}$ & $(0.003)$ & 34,599 \\
\hline Post-discharge complications of newborn & & & & & & \\
\hline $\begin{array}{l}\text { Breast feeding or drinking poorly (yes=1, } \\
\text { otherwise=0) }\end{array}$ & $0.004^{* * *}$ & $(0.000)$ & 36,692 & 0.002 & $(0.925)$ & 34,600 \\
\hline Fever or cold to touch (yes=1, otherwise=0) & $0.003^{* * *}$ & $(0.000)$ & 36,692 & $-0.031^{*}$ & $(0.064)$ & 34,600 \\
\hline Fast or difficult breathing (yes=1, otherwise=0) & $0.002^{* * *}$ & $(0.000)$ & 36,692 & $-0.027 * *$ & $(0.048)$ & 34,600 \\
\hline Blood in stool (yes=1, otherwise=0) & $0.000^{* *}$ & $(0.010)$ & 35,428 & -0.006 & $(0.402)$ & 33,403 \\
\hline
\end{tabular}

Note: Using primary samples (excluding home deliveries and PHSs above 500 hours) from the household and woman surveys of DLHS-4, the above table shows estimated values of $\hat{\rho}$ from running logit regressions of equations (1) and (4), which are nonIV and two stage IV regressions. Estimates of $\hat{\rho}$ are marginal effects. p-values estimated from robust standard errors are in parentheses. * $p<0.10, * * p<0.05, * * * p<0.01$.

We then run OLS regression of reduced form equation (2), and logit regressions of reduced form equation (3). Table (6) shows all of these regressions' results (e.g., marginal effects in the cases of logit regressions) with p-values (estimated from robust standard errors) in parentheses, except the parameters of state dummies and birth year dummies. Although we are mainly interested in the coefficients of the JSY dummy, we show the coefficients of the control variables here, as some might also be interested to know how much the control variables affect hours of PHS and the PDCs. In almost all cases, the coefficients of the JSY dummy and the control variables have expected signs. For example, in the case of hours of PHS, the JSY dummy has a negative coefficient, and there are positive coefficients of that dummy in the cases of all PDCs except poor breastfeeding to the newborn. So, we can expect that ILS and 2SLS estimates of the causal effects will also give us expected signs in all PDCs except poor breastfeeding to the newborn. However, we show results from 2 SLS only. 
Table 6: Results of reduced form equations

\begin{tabular}{|c|c|c|c|c|c|c|c|c|c|c|c|}
\hline & & \multicolumn{6}{|c|}{ Post-discharge complications of mother } & \multicolumn{4}{|c|}{ Post-discharge complications of newborn } \\
\hline & Hours & High & Abdominal & Vaginal & Excessive & & Severe & Breastfeeding & Fever & Difficult & Blood \\
\hline & of stay & fever & pain & discharge & bleeding & Convulsions & headache & \begin{tabular}{|l|} 
poorly \\
\end{tabular} & or cold & breathing & in stool \\
\hline \multirow[t]{2}{*}{ JSY } & $-2.861 * * *$ & 0.004 & $0.008 * * *$ & $0.003 *$ & $0.003 *$ & $0.003 * *$ & $0.006 * * *$ & -0.000 & $0.004 *$ & $0.003^{*}$ & 0.001 \\
\hline & $(0.000)$ & $(0.189)$ & $(0.005)$ & $(0.052)$ & $(0.083)$ & $(0.047)$ & $(0.005)$ & $(0.925)$ & $(0.073)$ & $(0.058)$ & $(0.418)$ \\
\hline \multicolumn{12}{|c|}{ Socioeconomic characteristics } \\
\hline \multirow[t]{2}{*}{ Wealth Index } & -0.166 & -0.001 & -0.000 & 0.000 & 0.000 & 0.000 & -0.001 & 0.000 & -0.001 & -0.000 & 0.000 \\
\hline & $(0.354)$ & $(0.270)$ & $(0.716)$ & $(0.900)$ & $(0.480)$ & $(0.132)$ & $(0.129)$ & $(0.763)$ & $(0.197)$ & $(0.341)$ & $(0.477)$ \\
\hline \multirow[t]{2}{*}{ Mother's age at birth } & $0.382 * * *$ & 0.000 & -0.000 & 0.000 & -0.000 & 0.000 & $-0.000^{* *}$ & -0.000 & -0.000 & $0.000 *$ & 0.000 \\
\hline & $(0.000)$ & $(0.852)$ & $(0.643)$ & $(0.519)$ & $(0.459)$ & $(0.307)$ & $(0.031)$ & $(0.131)$ & $(0.532)$ & $(0.080)$ & $(0.143)$ \\
\hline \multirow[t]{2}{*}{ Mother's education } & 0.165 & 0.000 & 0.000 & $0.001 * * *$ & 0.000 & -0.000 & 0.000 & 0.000 & 0.000 & -0.000 & -0.000 \\
\hline & $(0.110)$ & $(0.292)$ & $(0.300)$ & $(0.009)$ & $(0.332)$ & $(0.770)$ & $(0.182)$ & $(0.623)$ & $(0.881)$ & $(0.894)$ & $(0.390)$ \\
\hline \multirow[t]{2}{*}{ Husband's education } & -0.106 & $-0.001 * *$ & -0.000 & -0.000 & -0.000 & 0.000 & -0.000 & -0.000 & -0.000 & 0.000 & -0.000 \\
\hline & $(0.294)$ & $(0.019)$ & $(0.308)$ & $(0.405)$ & $(0.439)$ & $(0.565)$ & $(0.134)$ & $(0.750)$ & $(0.136)$ & $(0.853)$ & $(0.715)$ \\
\hline \multirow[t]{2}{*}{ Hindu } & $1.238 *$ & -0.003 & -0.004 & -0.001 & -0.003 & 0.001 & $-0.009 * * *$ & 0.002 & -0.000 & -0.003 & 0.001 \\
\hline & $(0.091)$ & $(0.276)$ & $(0.152)$ & $(0.546)$ & $(0.196)$ & $(0.265)$ & $(0.001)$ & $(0.475)$ & $(0.988)$ & $(0.143)$ & $(0.203)$ \\
\hline \multirow[t]{2}{*}{ Scheduled caste } & $1.827 * * *$ & $0.006^{* *}$ & 0.000 & -0.001 & 0.002 & 0.001 & $-0.004^{*}$ & $0.005^{*}$ & 0.002 & $0.004 * *$ & 0.001 \\
\hline & $(0.009)$ & $(0.035)$ & $(0.858)$ & $(0.640)$ & $(0.258)$ & $(0.540)$ & $(0.083)$ & $(0.082)$ & $(0.257)$ & $(0.036)$ & $(0.329)$ \\
\hline \multirow[t]{2}{*}{ Tribe } & $-2.676 * *$ & -0.006 & -0.006 & $-0.006 * *$ & $-0.009 * * *$ & $-0.004^{* *}$ & -0.004 & $-0.009 * *$ & 0.001 & -0.002 & 0.000 \\
\hline & $(0.022)$ & $(0.218)$ & $(0.201)$ & $(0.015)$ & $(0.003)$ & $(0.019)$ & $(0.320)$ & $(0.023)$ & $(0.709)$ & $(0.465)$ & $(0.892)$ \\
\hline \multirow[t]{2}{*}{ Poor } & -1.154 & 0.004 & 0.003 & 0.001 & -0.000 & 0.001 & 0.002 & $-0.005^{* *}$ & $-0.004 *$ & -0.000 & -0.001 \\
\hline & $(0.102)$ & $(0.107)$ & $(0.234)$ & $(0.614)$ & $(0.983)$ & $(0.540)$ & $(0.287)$ & $(0.043)$ & $(0.061)$ & $(0.932)$ & $(0.511)$ \\
\hline \multirow[t]{2}{*}{ Birth order } & $-1.170^{* * *}$ & 0.001 & $0.005^{* * *}$ & 0.000 & 0.001 & 0.000 & $0.003 * * *$ & $-0.003 * *$ & $-0.002^{*}$ & $-0.002 * * *$ & $-0.001^{*}$ \\
\hline & $(0.001)$ & $(0.392)$ & $(0.000)$ & $(0.763)$ & $(0.511)$ & $(0.549)$ & $(0.003)$ & $(0.043)$ & $(0.094)$ & $(0.008)$ & $(0.052)$ \\
\hline \multirow[t]{2}{*}{ Rural } & $1.410 * *$ & -0.001 & 0.001 & 0.001 & 0.001 & 0.001 & $-0.005 * *$ & 0.004 & 0.002 & 0.002 & 0.001 \\
\hline & $(0.019)$ & $(0.692)$ & $(0.793)$ & $(0.350)$ & $(0.684)$ & $(0.287)$ & $(0.020)$ & $(0.118)$ & $(0.265)$ & $(0.227)$ & $(0.170)$ \\
\hline \multicolumn{12}{|c|}{ Delivery time complications of mother } \\
\hline Premature labour & \begin{tabular}{l|l}
1.536 \\
\end{tabular} & $0.061 * * *$ & $0.051 * * *$ & $0.007 * * *$ & 0.004 & $0.007 * * *$ & $0.015 * * *$ & $0.041 * * *$ & $0.018 * * *$ & $0.014 * * *$ & $0.002 *$ \\
\hline & $(0.103)$ & $(0.000)$ & $(0.000)$ & $(0.001)$ & $(0.132)$ & $(0.001)$ & $(0.000)$ & $(0.000)$ & $(0.000)$ & $(0.000)$ & $(0.089)$ \\
\hline Excessive bleeding & $3.826^{* * *}$ & $0.040 * * *$ & $0.042 * * *$ & $0.031 * * *$ & $0.056^{* * * *}$ & $0.004 *$ & $0.014 * * *$ & $0.023 * * *$ & $0.029 * * *$ & $0.011 * * *$ & $0.006 * *$ \\
\hline & $(0.005)$ & $(0.000)$ & $(0.000)$ & $(0.000)$ & $(0.000)$ & $(0.085)$ & $(0.002)$ & $(0.000)$ & $(0.000)$ & $(0.002)$ & $(0.018)$ \\
\hline Prolonged labour & $2.775 * *$ & $0.027 * * *$ & $0.046 * * *$ & $0.027 * * *$ & $0.030 * * *$ & $0.006^{* * *}$ & $0.035 * * *$ & $0.022 * * *$ & $0.020 * * *$ & $0.011^{* * * *}$ & -0.000 \\
\hline & $(0.023)$ & $(0.000)$ & $(0.000)$ & $(0.000)$ & $(0.000)$ & $(0.009)$ & $(0.000)$ & $(0.000)$ & $(0.000)$ & $(0.001)$ & $(0.822)$ \\
\hline Obstructed labour & $-2.246 * *$ & $0.013 * * *$ & $0.031 * * *$ & $0.013 * * *$ & 0.019 *** & $0.011 * * *$ & $0.028 * * *$ & 0.005 & 0.001 & 0.003 & $0.003 *$ \\
\hline & $(0.021)$ & $(0.003)$ & $(0.000)$ & $(0.000)$ & $(0.000)$ & $(0.000)$ & $(0.000)$ & $(0.211)$ & $(0.788)$ & $(0.269)$ & $(0.084)$ \\
\hline Breech presentation & 0.989 & -0.003 & -0.003 & -0.000 & $0.011 *$ & $0.023 * * *$ & 0.001 & $0.013 *$ & -0.003 & $0.015 * *$ & 0.003 \\
\hline & $(0.688)$ & $(0.618)$ & $(0.680)$ & $(0.969)$ & $(0.055)$ & $(0.000)$ & $(0.851)$ & $(0.076)$ & $(0.479)$ & $(0.012)$ & $(0.221)$ \\
\hline Convulsion & $9.454 * * *$ & $0.091 * * *$ & $0.051 * * *$ & $0.015^{* * *}$ & $0.028 * * *$ & $0.040 * * *$ & $0.061 * * *$ & 0.030 *** & $0.035 * * *$ & $0.024 * * *$ & $0.008^{* * * *}$ \\
\hline & $(0.000)$ & $(0.000)$ & $(0.000)$ & $(0.001)$ & $(0.000)$ & $(0.000)$ & $(0.000)$ & $(0.000)$ & $(0.000)$ & $(0.000)$ & $(0.007)$ \\
\hline cesarean & $35.063 * * *$ & 0.013 & 0.006 & -0.001 & 0.000 & $-0.006 * * *$ & 0.011 & $0.018 * *$ & -0.004 & $0.010 *$ & $0.005^{*}$ \\
\hline & $(0.000)$ & $(0.111)$ & $(0.440)$ & $(0.744)$ & $(0.974)$ & $(0.004)$ & $(0.104)$ & $(0.035)$ & $(0.426)$ & $(0.075)$ & $(0.085)$ \\
\hline Health facility types & & & & & & & & & & & \\
\hline SHC & $-14.854 * * *$ & -0.004 & 0.005 & 0.005 & 0.002 & 0.007 & -0.001 & -0.007 & $-0.014 * * *$ & -0.004 & -0.002 \\
\hline & $(0.000)$ & $(0.634)$ & $(0.529)$ & $(0.298)$ & $(0.726)$ & $(0.156)$ & $(0.855)$ & $(0.334)$ & $(0.000)$ & $(0.285)$ & $(0.139)$ \\
\hline $\mathrm{PHC}$ & $-11.424 * * *$ & -0.003 & 0.005 & 0.002 & 0.004 & 0.001 & 0.000 & 0.006 & $-0.006^{* *} *$ & $-0.004 * *$ & -0.000 \\
\hline & $(0.000)$ & $(0.433)$ & $(0.227)$ & $(0.472)$ & $(0.219)$ & $(0.639)$ & $(0.937)$ & $(0.161)$ & $(0.020)$ & $(0.035)$ & $(0.946)$ \\
\hline $\mathrm{CHC}$ & $-11.327 * * *$ & -0.004 & -0.002 & -0.002 & -0.002 & 0.001 & -0.004 & 0.005 & -0.002 & -0.002 & $-0.002 *$ \\
\hline & $(0.000)$ & $(0.391)$ & $(0.610)$ & $(0.515)$ & $(0.617)$ & $(0.811)$ & $(0.289)$ & $(0.351)$ & $(0.533)$ & $(0.555)$ & $(0.058)$ \\
\hline SDH/DH & $-2.147 * * *$ & 0.002 & 0.003 & $0.003 *$ & -0.001 & 0.002 & 0.000 & -0.002 & -0.003 & $-0.005^{* * *}$ & -0.001 \\
\hline & $(0.002)$ & $(0.401)$ & $(0.237)$ & $(0.063)$ & $(0.723)$ & $(0.130)$ & $(0.953)$ & $(0.466)$ & $(0.144)$ & $(0.001)$ & $(0.519)$ \\
\hline Constant & $30.551 * * *$ & & & & & & & & & & \\
\hline & $(0.000)$ & & & & & & & & & & \\
\hline State dummies & yes & yes & yes & yes & yes & yes & yes & yes & yes & yes & yes \\
\hline Birth year dummies & yes & yes & yes & yes & yes & yes & yes & yes & yes & yes & yes \\
\hline$R^{2}$ & 0.176 & & & & & & & & & & \\
\hline$N$ & 34,602 & 34,599 & 34,599 & 34,599 & 34,599 & 34,502 & 34,599 & 34,600 & 34,600 & 34,600 & 33,403 \\
\hline
\end{tabular}

Note: Using primary samples (excluding home deliveries and PHSs above 500 hours) from the household and woman surveys of DLHS-4, this table shows regressions' results of equations (2) and (3) excluding coefficients of state and birth year dummies, which were also used in regressions. OLS regression results of hours of PHS are shown in column 2, and marginal effects estimated from logit regressions of PDCs are shown in the next ten columns. We do not report pseudo $R^{2}$ from logit regressions, as they are probably not important to show. p-values estimated from robust standard errors are in parentheses. $* p<0.10, * * p<0.05, * * * p<0.01$.

Following equation (4), we run logit regressions of all PDCs on the predicted value of $X_{i}$, 
$\hat{X}_{i}$, generated from the OLS regression of equation (2), and all control variables. These are 2SLS regressions. In IV columns of Table (5), we show marginal effects of $\hat{X}_{i} / 24$ on the probabilities of PDCs with p-values (estimated from robust standard errors) in parentheses. Results are expectedly negatives in all cases except poor breastfeeding to the newborn and statistically significant in most of the cases except high fever of mother, and poor breastfeeding to the newborn and blood in the stool of the newborn. The sizes of the results are reasonable. For example, if PHS increases by one day, the probability of abdominal pain after the discharge decreases by 0.062 or 6.2 percentage points.

\subsection{Results with subsamples}

\subsubsection{Summary statistics and the validity of the instrument}

As we have seen in Table (1), differences in means of almost all control variables between JSY mothers and nonJSY mothers are statistically and significantly different from zero. These violate the second identification condition of an IV technique. To satisfy the condition, following [3], we discard some observations based on the propensity score of the JSY dummy, $\operatorname{Pr}\left(J S Y_{i}=\right.$ $\left.1 \mid Z_{1 i}, Z_{2 i}, \ldots, Z_{k i}\right)=$ pscore, so that the control variables overlap between JSY mothers and nonJSY mothers, and in this way, differences in means of the control variables between the two groups of mothers may become zero. We run a logit regression of the JSY dummy on the control variables and then estimate pscore. We do not show results of that logit regression, as they are not important here. Figure (3) plots the densities of the propensity scores for two groups of women. We see that the control group (indicated by the solid yellow line, JSY $=0$ ) has the highest density at pscore close to 0 , which implies the violation of overlapping, and suggests to drop observations at lower values of $\operatorname{Pr}\left(J S Y_{i}=1 \mid Z_{1 i}, Z_{2 i}, \ldots, Z_{k i}\right)=$ pscore.

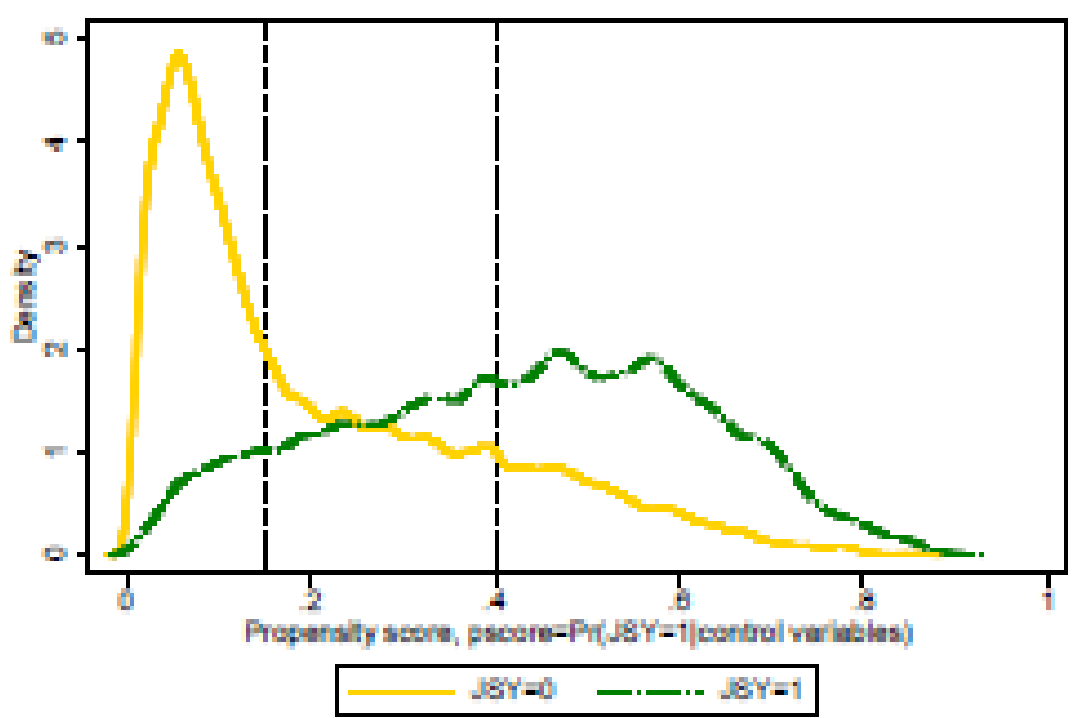

Note: Using primary samples (excluding home deliveries and PHSs above 500 hours) from the household and woman surveys of DLHS-4, we run logit regression (not shown) of the JSY dummy (1 yes, 0 no) on the control variables, and from that regression we estimate the propensity score, $\operatorname{Pr}\left(J S Y=1 \mid Z_{1 i}, Z_{2 i}, \ldots, Z_{k i}\right)$, for every woman/observation. In this figure, we plot densities of the propensity scores, estimated from the triangular kernel, for two groups of JSY (e.g., long-dashed and dotted green line for the treatment group of JSY and the solid yellow line for the control group of JSY). We drop observations outside of two dashed vertical lines at 0.15 and 0.40 , as densities mostly overlap between these two lines. In other words, means of the control variables between two groups of JSY will be close, and thus, the JSY instrument will be valid.

Figure 3: Plotting the densities of the propensity scores for two groups of women (JSY and 
nonJSY)

However, [3] suggested to drop observations outside of $0.10<$ pscore $<0.90$. We do accordingly and drop further observations outside of $0.15<$ pscore $<0.40$ (e.g., outside of two dashed vertical lines in Figure (3)). We select this range of pscore on an ad-hoc basis, as densities of pscore in the two groups of mothers mostly overlap within this range. We call $0.10<$ pscore $<0.90$ Sample1 and $0.15<$ pscore $<0.40$ Sample2. We drop further observations within $0.15<$ pscore $<0.40$, in which, we restrict samples to those births, which are the first since 2008 not only in women but also in their households. Thus, we choose the final subsample, named as Sample3. In this way, the second identification condition of an IV technique tends to be satisfied, as consequently the difference in means of the control variables become statistically insignificant between JSY and nonJSY mothers (see Table (7)). In Sample3, only two control variables have pvalues less than 0.01 , and all other control variables have very high p-values. These imply that the second identification condition is mostly satisfied. With this subsample, the first identification condition is also satisfied as F-value is 13 , which crosses the threshold of $F=10$ (stock2002survey). So, our results are more reliable with Sample3. After Table (8), all other Tables show results using Sample3. One may concern, as we lose many observations. However, the validity of the instrument is much more critical than losing observations. Otherwise, in the presence of the endogeneity problem, the results will remain biased even if sample size goes to infinity.

Table 7: Mean differences of variables by different subsamples based on the propensity score and birth order

\begin{tabular}{|c|c|c|c|c|c|c|}
\hline \multirow{2}{*}{$\frac{2^{*}}{(\mathrm{rl}) 2-3(\mathrm{rl}) 4-5(\mathrm{rl}) 6-7}$} & \multicolumn{2}{|c|}{ Sample1 } & \multicolumn{2}{|c|}{ Sample2 } & \multicolumn{2}{|c|}{ Sample3 } \\
\hline & Diff. & p-value & Diff. & $p$-value & Diff. & $\mathrm{p}$-value \\
\hline $\begin{array}{c}\text { Hours of postnatal } \\
\text { hospital stay }\end{array}$ & -2.471 & $(0.001)$ & -3.300 & $(0.004)$ & -5.961 & $(0.002)$ \\
\hline \multicolumn{7}{|l|}{$\begin{array}{c}\text { Post-discharge } \\
\text { complications of } \\
\text { mother }\end{array}$} \\
\hline $\begin{array}{l}\text { High fever }(\text { yes }=1, \\
\text { otherwise }=0 \text { ) }\end{array}$ & -0.000 & $(0.902)$ & 0.009 & $(0.080)$ & 0.014 & $(0.140)$ \\
\hline $\begin{array}{c}\text { Abdominal pain } \\
(\text { yes }=1 \text {, otherwise }=0)\end{array}$ & 0.013 & $(0.000)$ & 0.016 & $(0.002)$ & 0.029 & $(0.002)$ \\
\hline $\begin{array}{c}\text { Vaginal discharge } \\
(\text { yes }=1, \text { otherwise }=0)\end{array}$ & 0.005 & $(0.040)$ & 0.005 & $(0.138)$ & 0.012 & $(0.076)$ \\
\hline $\begin{array}{c}\text { Excessive bleeding } \\
(\mathrm{yes}=1 \text {, otherwise }=0)\end{array}$ & 0.004 & $\begin{array}{l}(0.098) \\
\end{array}$ & 0.004 & $(0.355)$ & -0.001 & $\begin{array}{l}(0.912) \\
\end{array}$ \\
\hline $\begin{array}{c}\text { Convulsions (yes }=1, \\
\text { otherwise }=0 \text { ) }\end{array}$ & 0.002 & $(0.320)$ & 0.007 & $(0.030)$ & 0.013 & $(0.026)$ \\
\hline $\begin{array}{c}\text { Severe headache } \\
(\text { yes }=1 \text {, otherwise }=0)\end{array}$ & 0.008 & $(0.005)$ & 0.008 & $(0.074)$ & 0.017 & $(0.037)$ \\
\hline \multicolumn{7}{|l|}{$\begin{array}{c}\text { Post-discharge } \\
\text { complications of } \\
\text { newborn }\end{array}$} \\
\hline $\begin{array}{c}\text { Breast feeding or } \\
\text { drinking poorly } \\
(\text { yes }=1 \text {, otherwise }=0)\end{array}$ & -0.003 & $(0.335)$ & -0.003 & $(0.456)$ & 0.004 & $(0.676)$ \\
\hline $\begin{array}{c}\text { Fever or cold to touch } \\
(\text { yes }=1 \text {, otherwise }=0)\end{array}$ & 0.002 & $(0.408)$ & 0.007 & $(0.109)$ & 0.028 & $(0.001)$ \\
\hline $\begin{array}{c}\text { Fast or difficult } \\
\text { breathing (yes }=1, \\
\text { otherwise }=0 \text { ) }\end{array}$ & 0.003 & $(0.178)$ & 0.003 & $(0.406)$ & 0.009 & $(0.155)$ \\
\hline $\begin{array}{c}\text { Blood in stool (yes }=1, \\
\text { otherwise }=0)\end{array}$ & 0.000 & $\begin{array}{l}(0.848) \\
\end{array}$ & 0.001 & $(0.730)$ & 0.005 & $\begin{array}{l}(0.197) \\
\end{array}$ \\
\hline Socioeconomic & & & & & & \\
\hline
\end{tabular}




\begin{tabular}{|c|c|c|c|c|c|c|}
\hline conditions & & & & & & \\
\hline Wealth index & -0.381 & $(0.000)$ & -0.063 & $(0.094)$ & -0.023 & $(0.740)$ \\
\hline $\begin{array}{l}\text { Mother's age at birth } \\
\text { (in year) }\end{array}$ & -0.399 & $(0.000)$ & -0.003 & $(0.974)$ & -0.025 & $(0.865)$ \\
\hline $\begin{array}{l}\text { Years of education } \\
\text { completed by mother }\end{array}$ & -0.464 & $(0.000)$ & -0.091 & $(0.204)$ & 0.006 & $(0.960)$ \\
\hline $\begin{array}{l}\text { Years of education } \\
\text { completed by husband }\end{array}$ & -0.598 & $(0.000)$ & 0.023 & $(0.757)$ & -0.016 & $(0.899)$ \\
\hline $\begin{array}{c}\text { Hindu (yes }=1, \\
\text { otherwise }=0 \text { ) }\end{array}$ & -0.028 & $(0.000)$ & -0.012 & $(0.234)$ & -0.059 & $(0.001)$ \\
\hline $\begin{array}{c}\text { Scheduled caste } \\
(\text { yes }=1, \text { otherwise }=0)\end{array}$ & 0.028 & $(0.000)$ & 0.019 & $(0.041)$ & -0.004 & $(0.792)$ \\
\hline $\begin{array}{l}\text { Tribe }(\text { yes }=1 \\
\text { otherwise }=0)\end{array}$ & 0.070 & $(0.000)$ & 0.008 & $(0.286)$ & 0.057 & $(0.000)$ \\
\hline $\begin{array}{l}\text { Poor }(\text { yes }=1, \\
\text { otherwise }=0)\end{array}$ & 0.084 & $(0.000)$ & 0.026 & $(0.009)$ & -0.019 & $(0.254)$ \\
\hline Birth order & -0.107 & $(0.000)$ & 0.026 & $(0.176)$ & 0.000 & (.) \\
\hline $\begin{array}{l}\text { Rural (yes }=1 \text {, } \\
\text { otherwise }=0 \text { ) }\end{array}$ & 0.057 & $(0.000)$ & 0.002 & $(0.825)$ & -0.012 & $(0.513)$ \\
\hline \multicolumn{7}{|l|}{$\begin{array}{c}\text { Delivery time } \\
\text { complications }\end{array}$} \\
\hline $\begin{array}{c}\text { Premature labour } \\
(\text { yes }=1, \text { otherwise }=0)\end{array}$ & -0.007 & $(0.080)$ & -0.004 & $(0.597)$ & -0.002 & $(0.873)$ \\
\hline $\begin{array}{l}\text { Excessive bleeding } \\
(\text { yes }=1 \text {, otherwise }=0)\end{array}$ & 0.000 & $(0.969)$ & -0.001 & $(0.779)$ & 0.008 & $(0.348)$ \\
\hline $\begin{array}{c}\text { Prolonged labour } \\
(\text { yes }=1 \text {, otherwise }=0)\end{array}$ & 0.011 & $(0.003)$ & 0.000 & $(0.944)$ & 0.011 & $(0.319)$ \\
\hline $\begin{array}{c}\text { Obstructed labour } \\
(\mathrm{yes}=1, \text { otherwise }=0)\end{array}$ & 0.001 & $(0.802)$ & -0.002 & $(0.702)$ & 0.006 & $(0.604)$ \\
\hline $\begin{array}{c}\text { Breech presentation } \\
(\text { yes }=1 \text {, otherwise }=0)\end{array}$ & -0.003 & $(0.082)$ & -0.002 & $(0.498)$ & 0.003 & $(0.524)$ \\
\hline $\begin{array}{c}\text { Convulsions (yes }=1, \\
\text { otherwise }=0 \text { ) }\end{array}$ & 0.004 & $(0.106)$ & -0.002 & $(0.552)$ & 0.001 & $(0.853)$ \\
\hline $\begin{array}{c}\text { cesarean }(y e s=1, \\
\text { otherwise }=0)\end{array}$ & -0.000 & $(0.938)$ & 0.005 & $(0.086)$ & 0.006 & $(0.392)$ \\
\hline \multicolumn{7}{|l|}{ Health facility type } \\
\hline $\begin{array}{c}\text { Sub-health center } \\
(\text { yes }=1 \text {, otherwise }=0)\end{array}$ & 0.003 & $(0.121)$ & 0.005 & $(0.224)$ & -0.001 & $(0.809)$ \\
\hline $\begin{array}{l}\text { Primary health center } \\
(\mathrm{yes}=1, \text { otherwise }=0)\end{array}$ & 0.061 & $(0.000)$ & 0.014 & $(0.062)$ & 0.003 & $(0.761)$ \\
\hline $\begin{array}{c}\text { Community health } \\
\text { center }(\text { yes }=1, \\
\text { otherwise }=0 \text { ) }\end{array}$ & 0.031 & $(0.000)$ & 0.011 & $(0.049)$ & 0.010 & $(0.227)$ \\
\hline $\begin{array}{c}\text { Sub-district or District } \\
\text { hospital (yes }=1 \\
\text { otherwise }=0 \text { ) }\end{array}$ & 0.059 & $(0.000)$ & 0.004 & $(0.689)$ & 0.018 & $(0.334)$ \\
\hline$N$ & 24,564 & & 11,155 & & 3,858 & \\
\hline
\end{tabular}

Note: Using the household and woman surveys of DLHS-4, the above table shows differences (Diff.) in means of variables between JSY recipients and nonrecipients of JSY, and p-values of these differences estimated from robust standard errors, for three different subsamples selected based on the propensity score and birth order. Sample1 includes samples with $0.10<$ pscore $<0.90$, Sample 2 includes samples with $0.15<$ pscore $<0.40$, and Sample3 includes samples with $0.15<$ pscore $<0.40$ and those women whose last births are the first births in their households.

\subsubsection{Regression results}

In Table (8), we show regression results for that three subsamples, Sample1, Sample2, and Sample3. We show only key results, which are $\hat{\gamma}$, estimated from the OLS regression of equation (2), and $\hat{\rho}$ (e.g., marginal effects), estimated from logit regressions of equations (1) and (4). The values of $\hat{\gamma}$ in Table (8) imply that the effect of the JSY dummy on hours of PHS becomes higher 
(in absolute terms) with the subsequent drops of observations. The values of $\hat{\rho}$ slightly reduce (in absolute terms) in most of the cases, with those drops. Again, we ignore the results under the nonIV case (results from equation (1)). When we reach our final subsample (Sample3), all values of $\hat{\rho}$ under the IV case become negatives, but they are statistically significant in the cases of abdominal pain, vaginal discharge, convulsion and severe headache of a mother, and fever or cold of the newborn.

Table 8: Estimates of key parameters, $\hat{\gamma}, \hat{\rho}$ (NonIV) and $\hat{\rho}$ (IV) from running regressions of equations (2), (1) and (4) respectively

\begin{tabular}{|c|c|c|c|c|c|c|c|}
\hline & & \multicolumn{2}{|c|}{ Sample1 $(N=24,564)$} & \multicolumn{2}{|c|}{ Sample2 $(N=11,155)$} & \multicolumn{2}{|c|}{ Sample3 $(N=3,858)$} \\
\hline & & Estimate & $p$-value & Estimate & p-value & Estimate & p-value \\
\hline & $\hat{\gamma}$ & $-2.798 * * *$ & $(0.000)$ & $-3.652 * * *$ & $(0.000)$ & $-6.910 * * *$ & $(0.000)$ \\
\hline \multicolumn{8}{|c|}{ Post-discharge complications of mother } \\
\hline \multirow[t]{2}{*}{ High fever } & $\hat{\rho}$ (NonIV) & $0.002 * * *$ & $(0.000)$ & 0.001 & $(0.175)$ & 0.000 & $(0.990)$ \\
\hline & $\hat{\rho}(\mathrm{IV})$ & -0.016 & $(0.522)$ & $-0.050^{*}$ & $(0.077)$ & -0.038 & $(0.108)$ \\
\hline \multirow[t]{2}{*}{ Abdominal pain } & $\hat{\rho}$ (NonIV) & $0.003 * * *$ & $(0.000)$ & $0.003 * * *$ & $(0.000)$ & $0.002 * *$ & $(0.011)$ \\
\hline & $\hat{\rho}(\mathrm{IV})$ & $-0.069 * * *$ & $(0.005)$ & $-0.081 * * *$ & $(0.001)$ & $-0.067 * * *$ & $(0.000)$ \\
\hline \multirow[t]{2}{*}{ Vaginal discharge } & $\hat{\rho}$ (NonIV) & 0.000 & $(0.106)$ & 0.000 & $(0.794)$ & 0.000 & $(0.832)$ \\
\hline & $\hat{\rho}(\mathrm{IV})$ & -0.023 & $(0.104)$ & -0.020 & $(0.181)$ & $-0.023^{*}$ & $(0.067)$ \\
\hline \multirow[t]{2}{*}{ Excessive bleeding } & $\hat{\rho}$ (NonIV) & $0.001 * * *$ & $(0.000)$ & 0.001 & $(0.360)$ & $0.002 *$ & $(0.064)$ \\
\hline & $\hat{\rho}(\mathrm{IV})$ & -0.020 & $(0.279)$ & -0.024 & $(0.201)$ & -0.000 & $(0.992)$ \\
\hline \multirow[t]{2}{*}{ Convulsion } & $\hat{\rho}$ (NonIV) & 0.000 & $(0.116)$ & -0.000 & $(0.976)$ & 0.000 & $(0.725)$ \\
\hline & $\hat{\rho}(\mathrm{IV})$ & $-0.020^{*}$ & $(0.062)$ & $-0.032 * * *$ & $(0.004)$ & $-0.023 * * *$ & $(0.002)$ \\
\hline \multirow[t]{2}{*}{ Severe headache } & $\hat{\rho}$ (NonIV) & $0.001 * * *$ & $(0.001)$ & 0.001 & $(0.411)$ & 0.001 & $(0.433)$ \\
\hline & $\hat{\rho}(\mathrm{IV})$ & $-0.058 * * *$ & $(0.003)$ & $-0.044 * *$ & $(0.032)$ & $-0.044 * * *$ & $(0.005)$ \\
\hline \multicolumn{8}{|c|}{ Post-discharge complications of newborn } \\
\hline \multirow[t]{2}{*}{ Breast feeding poorly } & $\hat{\rho}$ (NonIV) & $0.004 * * *$ & $(0.000)$ & $0.003 * * *$ & $(0.000)$ & $0.005 * * *$ & $(0.000)$ \\
\hline & $\hat{\rho}(\mathrm{IV})$ & 0.021 & $(0.357)$ & 0.016 & $(0.528)$ & -0.000 & $(0.986)$ \\
\hline \multirow[t]{2}{*}{ Fever or cold } & $\hat{\rho}$ (NonIV) & $0.003 * * *$ & $(0.000)$ & 0.002 *** & $(0.000)$ & $0.003^{* * *}$ & $(0.000)$ \\
\hline & $\hat{\rho}(\mathrm{IV})$ & -0.027 & $(0.138)$ & $-0.040 * *$ & $(0.037)$ & $-0.063 * * *$ & $(0.000)$ \\
\hline \multirow[t]{2}{*}{ Difficult breathing } & $\hat{\rho}$ (NonIV) & $0.002 * * *$ & $(0.000)$ & $0.001 * * *$ & $(0.000)$ & $0.002 * * *$ & $(0.003)$ \\
\hline & $\hat{\rho}(\mathrm{IV})$ & -0.020 & $(0.183)$ & -0.015 & $(0.306)$ & -0.018 & $(0.172)$ \\
\hline \multirow[t]{2}{*}{ Blood in stool } & $\hat{\rho}$ (NonIV) & 0.000 & $(0.335)$ & -0.000 & $(0.291)$ & -0.000 & $(0.590)$ \\
\hline & $\hat{\rho}(\mathrm{IV})$ & -0.005 & $(0.435)$ & -0.002 & $(0.771)$ & -0.011 & $(0.237)$ \\
\hline
\end{tabular}

Note: Using the household and woman surveys of DLHS-4, for three different subsamples, the above table shows estimated values of key parameters, $\hat{\gamma}, \hat{\rho}$ (NonIV) and $\hat{\rho}$ (IV) from running regressions of equations (2), (1) and (4) respectively and their p-values estimated from the robust standard errors in parentheses. Estimates of $\hat{\rho}$ are marginal effects from logit regressions. $* p<0.10, * * p<0.05, * * * p<0.01$.

\subsection{Joint Randomization Test}

As is seen in the last two columns (Sample3) of Table (7), means of only two control variables (Hindu and Tribe dummies) are statistically and significantly different between JSY recipients and nonrecipients of JSY. When means of most of the control variables are statistically and insignificantly different between two groups of JSY, statistically significant mean difference in the case of two variables may happen due to random chance. They might not cause any problem to the overall random distribution of the control variables and hence may not violate the second identification condition of IV regressions. In the case of Regression Discontinuity Design, [8] suggested running joint randomization test by running seemingly unrelated regression (SUR) model. Following them, using the final subsample (Sample3), we jointly regress all control variables listed in Table (7) on JSY dummy using SUR model with the assumption of correlated errors among regression equations. The chi-square value is 36.54 and p-value is 0.064 , which reject the null hypothesis that the coefficients of JSY are jointly zero, at 10\% level. In another word, joint 
randomization of the control variables exists at $5 \%$ level, not at $10 \%$ level. Although there is a weak evidence of joint randomization, which is supposed to cause some biases in our final results if the control variables have effects on post-discharge complications, we expect that such biases will be very low and they will not make the significant negative relationships between PHS and the PDCs disappear. However, the control variables are added in both first stage and second stage regressions, and our final results come after controlling for their effects. Moreover, we run the SUR model for the PDCs (jointly regress all of them on JSY dummy and the control variables) and test the joint hypothesis that the coefficients of JSY dummy are jointly zero. ${ }^{10}$ We find that pvalue is less than 0.001 . We can say that our results are robust, and the weak randomization does not threaten the validity of our final results.

\subsection{Falsification Test with Morbidities at the Time of Pregnancy}

As another robustness check, we also do a falsification test. It is practical to assume that PHS does not affect pregnancy time morbidities. The violation of the assumption will indicate that our final results of the negative relationships between PHS and the PDCs are spurious. Using logit model, we first separately regress each pregnancy time morbidity on the predicted PHS (estimated from the first stage regression) and the control variables. Table (9) shows the marginal effects of the predicted PHS. We see that the assumption is violated in the case of three morbidities paleness, abnormal position of the fetus and excessive vomiting. Considering simultaneous equations, we then regress all morbidities on the same variables using SUR model and test the joint null hypothesis that the coefficients of the predicted PHS are together zero. The p-value is 0.21, and we cannot reject the joint null. Similarly, we do the joint test for the PDCs. The p-value is less than 0.001. We infer that PHS has overall effects on the PDCs but not on the pregnancy time morbidities, and our final results are not spurious.

Table 9: The effects of PHS on the pregnancy time morbidities (IV regressions)

\begin{tabular}{|l|c|c|}
\hline Pregnancy time Morbidities & IV Estimate & p-value \\
\hline Swelling of hands feet and face & 0.024 & $(0.619)$ \\
\hline Paleness/giddiness/weakness & $-0.057^{*}$ & $(0.087)$ \\
\hline Visual disturbance & -0.005 & $(0.738)$ \\
\hline Excessive fatigue & -0.026 & $(0.460)$ \\
\hline Convulsion & 0.021 & $(0.145)$ \\
\hline Weak or no movement of fetus & -0.015 & $(0.308)$ \\
\hline Abnormal position of fetus & $-0.027^{* * *}$ & $(0.001)$ \\
\hline Malaria & 0.009 & $(0.317)$ \\
\hline Excessive vomiting & $-0.107^{* *}$ & $(0.043)$ \\
\hline High blood pressure & -0.028 & $(0.122)$ \\
\hline Jaundice & 0.010 & $(0.335)$ \\
\hline Excessive bleeding & -0.002 & $(0.867)$ \\
\hline Vaginal discharge & -0.004 & $(0.605)$ \\
\hline$N$ & 3,858 & \\
\hline
\end{tabular}

Note: Using the final subsample (Sample3) from the household and woman surveys of DLHS-4, we run two stage IV regressions of all above morbidities as similar ways as we did for PDCs. The above table shows the coefficients of the predicted days of PHS (e.g., marginal effects from logit regressions). p-values estimated from robust standard errors are in parentheses. $* p<0.10, * * p<0.05$, $* * * p<0.01$. In only three morbidities, results are statistically significant, but if we run the second stage IV regressions of all above morbidities together in a seemingly unrelated regression

${ }_{10}$ In the case of control variables, we expect that the joint null hypothesis will not be rejected, but we hope the opposite in the fact of postdischarge complications. Another issue is that given that the coefficient of JSY in the first stage regression is significant, significant coefficients of JSY in the regressions of the reduced form equations of PDCs will ensure the significant coefficients of the predicted PHS in the second stage regressions. 
model, we find that the coefficients of the predicted days of PHS in all morbidities are not jointly significant. These indicate that our causal estimates (results under IV cases in Table (8)) are not spurious.

\subsection{Heterogenous Effects}

The negative relationship between PHS and the PDCs might not be linear. As hours of PHS goes higher, the sizes of negative results can be smaller (in absolute terms). Because who stay longer their small stay gaps may not cause any changes in PDCs, but small stay gaps among low stayers can make substantial changes in PDCs. Usually, women with cesarean deliveries stay longer, but vaginal deliveries require a shorter stay. In our data, the average hours of stay after vaginal deliveries is 60 , and it becomes 153 after cesarean deliveries. To check the nonlinearity, we divide hours of PHS into two parts - less than 72 hours takes bottom 50\% women, and greater than or equal to 72 takes top $50 \%$ women. Using the final subsample, Table (10) shows causal estimates (the estimated values of $\hat{\rho}$ from IV regressions; e.g., marginal effects from logit regressions of the second stage) along with the estimated values of $\hat{\gamma}$. In the first group (low stayers), the average stay gap between JSY recipients and nonrecipients of JSY is around -2 hours, and it is around -12 hours in the second group. The negative causal effects are highly significant in most of the cases of the former group, but the latter group has a significant result in only one case - severe headache. Results clearly indicate that there is a nonlinearity between PHS and PDCs.

Results may also vary by ethnicity. Table (11) shows that women with nonscheduled caste, nontribe, and nonpoor status have statistically significant results in higher cases of PDCs compared to their counterparts. The possible reason is that a higher proportion of them goes to private hospitals where stay differences may cause high effects on PDCs. On the other hand, women with scheduled caste, tribe, and poor status go to public hospitals in a higher proportion. As most of the government hospitals lack in the quality service including the shortage of professional medical staff, medicines and other facilities, stays gaps there may have small or no effects on PDCs.

Table 10: Estimates of key parameters, $\hat{\gamma}$ and $\hat{\rho}$ (IV) by hours of stay

\begin{tabular}{|c|c|c|c|c|}
\hline & \multicolumn{2}{|c|}{$\begin{array}{l}\text { Hours of Stay<72 } \\
\quad(N=1,905)\end{array}$} & \multicolumn{2}{|c|}{$\begin{array}{l}\text { Hours of Stay } \geq 72 \\
\quad(N=1,953)\end{array}$} \\
\hline & Estimate & p-value & Estimate & p-value \\
\hline$\hat{\gamma}$ & $-1.923 * *$ & $(0.018)$ & $-12.129 * * *$ & $(0.000)$ \\
\hline \multicolumn{5}{|c|}{ Post-discharge complications of mother $(\widehat{\rho}(I V))$} \\
\hline High fever & $-0.261 * *$ & $(0.020)$ & 0.005 & $(0.821)$ \\
\hline Abdominal pain & $-0.282 * * *$ & $(0.000)$ & -0.014 & $(0.448)$ \\
\hline Vaginal discharge & $-0.129 * *$ & $(0.045)$ & -0.005 & $(0.663)$ \\
\hline Excessive bleeding & -0.004 & $(0.959)$ & 0.005 & $(0.743)$ \\
\hline Convulsions & $-0.140 * * *$ & $(0.007)$ & -0.004 & $(0.356)$ \\
\hline Severe headache & $-0.125 *$ & $(0.066)$ & $-0.025^{*}$ & $(0.067)$ \\
\hline \multicolumn{5}{|c|}{ Post-discharge complications of newborn $(\widehat{\rho}(I V))$} \\
\hline Breast feeding poorly & -0.137 & $(0.159)$ & 0.033 & $(0.104)$ \\
\hline Fever or cold & $-0.267 * * *$ & $(0.001)$ & -0.020 & $(0.161)$ \\
\hline Difficult breathing & -0.096 & $(0.142)$ & -0.005 & $(0.638)$ \\
\hline Blood in stool & -0.041 & $(0.393)$ & -0.009 & $(0.130)$ \\
\hline
\end{tabular}

Note: We split the final subsample (Sample3) from the household and woman surveys of DLHS-4 into two. If PHS is less than 72 hours, the sample size is 1,905 (around 50\% of Sample3), and for PHS above or equal to 72 hours, the sample size is 1,953 (another 50\%). For these two split 
subsamples of Sample3, the above table shows estimated values of key parameters, $\hat{\gamma}$ and $\hat{\rho}$ (IV) from running regressions of equations (2) and (4) respectively and their p-values estimated from the robust standard errors in parentheses. Estimates of $\hat{\rho}$ are marginal effects from logit regressions. $* p<0.10, * * p<0.05, * * * p<0.01$. As the negative causal estimates are more apparent in lower stays than that in the longer stays, there is a nonlinear relationship between PHS and PDCs.

Table 11: Estimates of key parameters, $\hat{\gamma}$ and $\hat{\rho}$ (IV) by ethnicity

\begin{tabular}{|c|c|c|c|c|c|c|}
\hline & \multicolumn{2}{|c|}{ Scheduled Caste } & \multicolumn{2}{|c|}{ Tribe } & \multicolumn{2}{|c|}{ Poor } \\
\hline & Yes $(N=933)$ & No $(N=2,925)$ & Yes $(N=495)$ & No $(N=3,363)$ & Yes $(N=1,027)$ & $\mathrm{No}(N=2,831)$ \\
\hline \multirow[t]{2}{*}{$\hat{y}$} & $-7.749 * *$ & $-6.680 * * *$ & $-9.395 * *$ & $-6.080 * * *$ & -3.202 & $-7.696 * * *$ \\
\hline & $(0.032)$ & $(0.001)$ & $(0.045)$ & $(0.002)$ & $(0.453)$ & $(0.000)$ \\
\hline \multicolumn{7}{|c|}{ Post-discharge complications of mother $(\widehat{\rho}(I V))$} \\
\hline \multirow[t]{2}{*}{ High fever } & 0.017 & $-0.056^{* *}$ & $-0.032 *$ & -0.033 & -0.136 & -0.025 \\
\hline & $(0.740)$ & $(0.025)$ & $(0.052)$ & $(0.278)$ & $(0.243)$ & $(0.243)$ \\
\hline \multirow[t]{2}{*}{ Abdominal pain } & -0.037 & $-0.076 * * *$ & -0.020 & $-0.059 * *$ & -0.116 & $-0.061 * * *$ \\
\hline & $(0.419)$ & $(0.000)$ & $(0.123)$ & $(0.014)$ & $(0.205)$ & $(0.001)$ \\
\hline \multirow[t]{2}{*}{ Vaginal discharge } & -0.014 & $-0.025^{*}$ & -0.007 & -0.022 & -0.043 & -0.019 \\
\hline & $(0.658)$ & $(0.074)$ & $(0.700)$ & $(0.174)$ & $(0.546)$ & $(0.143)$ \\
\hline \multirow[t]{2}{*}{ Excessive bleeding } & -0.034 & 0.008 & 0.013 & -0.007 & 0.132 & -0.015 \\
\hline & $(0.284)$ & $(0.712)$ & $(0.544)$ & $(0.743)$ & $(0.206)$ & $(0.360)$ \\
\hline \multirow[t]{2}{*}{ Convulsions } & $-0.061 * *$ & $-0.018 * *$ & -0.001 & $-0.032 * * *$ & -0.039 & $-0.022 * *$ \\
\hline & $(0.036)$ & $(0.045)$ & $(0.624)$ & $(0.001)$ & $(0.162)$ & $(0.016)$ \\
\hline \multirow[t]{2}{*}{ Severe headache } & $\begin{array}{l}-0.019 \\
\end{array}$ & $-0.049 * * *$ & $\begin{array}{l}-0.026 \\
\end{array}$ & $-0.041 * *$ & -0.115 & $-0.040 * * *$ \\
\hline & $(0.473)$ & $(0.008)$ & $(0.232)$ & $(0.030)$ & $(0.268)$ & $(0.006)$ \\
\hline \multicolumn{7}{|c|}{ Post-discharge complications of newborn $(\widehat{\rho}(I V))$} \\
\hline \multirow[t]{2}{*}{ Breast feeding poorly } & 0.026 & -0.001 & 0.046 & -0.016 & 0.178 & -0.016 \\
\hline & $(0.635)$ & $(0.959)$ & $(0.320)$ & $(0.565)$ & $(0.116)$ & $(0.522)$ \\
\hline \multirow[t]{2}{*}{ Fever or cold } & $-0.062 * *$ & $-0.058 * * *$ & 0.004 & $-0.076 * * *$ & $-0.116 * *$ & $-0.049 * * *$ \\
\hline & $(0.046)$ & $(0.002)$ & $(0.849)$ & $(0.000)$ & $(0.036)$ & $(0.008)$ \\
\hline \multirow[t]{2}{*}{ Difficult breathing } & 0.007 & -0.018 & 0.000 & -0.022 & -0.074 & -0.008 \\
\hline & $\begin{array}{l}(0.799) \\
\end{array}$ & $(0.232)$ & $(0.880)$ & (0.209) & $(0.140)$ & $(0.557)$ \\
\hline \multirow[t]{2}{*}{ Blood in stool } & 0.001 & -0.014 & -0.000 & -0.007 & 0.000 & $\begin{array}{l}-0.004 \\
\end{array}$ \\
\hline & $(0.799)$ & $(0.123)$ & $(0.637)$ & $(0.445)$ & $(0.177)$ & $(0.393)$ \\
\hline
\end{tabular}

Note: Here we split the final subsample (Sample3) from the household and woman surveys of DLHS-4 into ethnic groups - scheduled caste ( $N=933)$, nonscheduled caste $(N=2,925)$, Tribe $(N=495)$, nontribe $(N=3,363)$, poor $(N=1,027)$, and nonpoor $(N=2,831)$. For these ethnic groups of Sample3, the above table shows estimated values of key parameters, $\hat{\gamma}$ and $\hat{\rho}$ (IV) from running regressions of equations (2) and (4) respectively and their $\mathrm{p}$-values estimated from the robust standard errors in parentheses. Estimates of $\hat{\rho}$ are marginal effects from logit regressions. * $p<0.10$, ${ }^{* *} p<0.05,{ }^{* * *} p<0.01$. Results imply that nonscheduled caste, nontribe and nonpoor are more affected by the stay than their counterparts.

\subsection{Results from Natural Grouping}

Now, we assume that both JSY and nonJSY women have similar socio-economic conditions in each of three natural groups, Scheduled Caste, Tribe and Poor with below poverty line card. Table (12) shows the main results under three groups. We do not drop any observations based on the propensity scores. Therefore, our results and sample sizes here will be different than those in Table (11). In Table (12), we see that the first identification condition does not hold in the case of Scheduled Caste, as the value of $\hat{\gamma}$ is statistically insignificant. In the other two natural groups, this condition is satisfied. However, the second identification condition (e.g., balancing 
covariates between JSY and nonJSY women) does hold in none of these three groups (we do not show covariates balancing tests). So, the results in Table (12) are not reliable.

Table 12: Estimates of key parameters, $\hat{\gamma}$ and $\hat{\rho}$ (IV) by three ethnic/natural groups

\begin{tabular}{|c|c|c|c|c|c|c|c|c|c|}
\hline & \multicolumn{3}{|c|}{ Scheduled Caste } & \multicolumn{3}{|c|}{ Tribe } & \multicolumn{3}{|c|}{ Poor } \\
\hline & Estimate & p-value & $N$ & Estimate & p-value & $N$ & Estimate & p-value & $N$ \\
\hline & & & & & & & & & \\
\hline$\hat{y}$ & -1.765 & $(0.194)$ & 8,055 & $-3.384 * *$ & $(0.028)$ & 4,929 & $-2.631 * *$ & $(0.019)$ & 11,197 \\
\hline \multicolumn{10}{|c|}{ Post-discharge complications of mother $(\widehat{\rho}(I V))$} \\
\hline High fever & 0.018 & $(0.818)$ & 8,013 & 0.002 & $(0.961)$ & 4,891 & 0.053 & $(0.212)$ & 11,188 \\
\hline Abdominal pain & -0.088 & $(0.192)$ & 8,017 & -0.064 & $(0.137)$ & 4,862 & -0.051 & $(0.229)$ & 11,133 \\
\hline Vaginal discharge & -0.020 & $(0.562)$ & 7,930 & -0.011 & $(0.661)$ & 4,820 & -0.007 & $(0.741)$ & 11,142 \\
\hline Excessive bleeding & -0.060 & $(0.227)$ & 7,948 & -0.018 & $(0.477)$ & 4,859 & 0.009 & $(0.767)$ & 10,970 \\
\hline Convulsions & -0.028 & $(0.340)$ & 7,724 & 0.011 & $(0.465)$ & 4,724 & 0.011 & $(0.542)$ & 10,846 \\
\hline Severe headache & -0.073 & $(0.163)$ & 8,037 & -0.010 & $(0.751)$ & 4,877 & $-0.074 * *$ & $(0.027)$ & 11,178 \\
\hline \multicolumn{10}{|c|}{ Post-discharge complications of newborn $(\widehat{\rho}(I V))$} \\
\hline Breast feeding poorly & 0.013 & $(0.831)$ & 8,051 & 0.041 & $(0.311)$ & 4,860 & $0.083 * *$ & $(0.017)$ & 11,181 \\
\hline Fever or cold & -0.045 & $(0.342)$ & 8,018 & -0.017 & $(0.677)$ & 4,907 & -0.037 & $(0.171)$ & 11,012 \\
\hline Difficult breathing & $-0.094 * *$ & $(0.021)$ & 8,018 & -0.007 & $(0.820)$ & 4,722 & -0.010 & $(0.666)$ & 11,054 \\
\hline Blood in stool & 0.001 & $(0.959)$ & 7,219 & 0.002 & $(0.917)$ & 3,862 & 0.008 & $(0.301)$ & 10,767 \\
\hline
\end{tabular}

Note: Here we think that women will be similar within ethnic groups. For the three ethnic groups, the above table shows estimated values of key

parameters, $\hat{\gamma}$ and $\hat{\rho}$ (IV) from running regressions of equations (2) and (4) respectively and their p-values estimated from the robust standard

errors in parentheses. Estimates of $\hat{\rho}$ are marginal effects from logit regressions. $* p<0.10, * * p<0.05, * * *<0.01$.

\subsection{Results from Nationally Representative Data}

All previous analyses were restricted to high performing states (HPS). One might be interested to know how much results are also in low performing states (LPS) and the national level. Using the nationally representative data, the fourth/latest round of National Family Health Survey (NFHS-4), we estimate the main results using the same models as we did for DLHS-4. Although JSY is a universal program in LPS, our IV strategy will remain the same in LPS. If we were interested in estimating the effects of JSY on maternal health outcomes, we would have used different models for LPS and HPS.

NFHS-4 is a large cross-section survey collected data on around 1.3 million reproductiveaged women (e.g., 15-49). Among them, approximately a half million women are from HPS, and the rest are from LPS. However, if we restrict the sample to those women who gave birth after 2006 and whose hours of stay are less than 500 as we did in DLHS-4, the sample size drastically drops to around 144,500 women (40\% from HPS and the rest from LPS). We have used the same covariates in regressions as we used in the previous analyses. The sample size further drops in regressions, as the common sample size of those covariates is around one hundred thousand (approximately 45\% from HPS).

Not all post-discharge complications in DLHS-4 are available in NFHS-4. There are only four post-discharge complications in NFHS-4. Two complications of mother/woman, high fever and vaginal discharge, are also available in DLHS-4. Among the two complications of the newborn, blood in stool is also available in DLHS-4, but diarrhoea is not there. Table (13) shows the key results for HPS, LPS and national. It should be noted that, as we did under Sample 3 in Table (8), for each of three categories, we have chosen closer JSY and nonJSY women in socioeconomic conditions based on a range of the propensity score and then have estimated key results. 
In this way, although we lose some further observations, we have been able to hold the second identification condition (we do not show propensity scores and covariates balancing tests). ${ }^{11}$ After maintaining this condition, the first identification condition is also satisfied in each case, as we see the values of $\hat{\gamma}$ are significantly negative. As we saw under Sample 3 in Table (8), high fever and excessive bleeding have again statistically insignificant results (e.g., IV estimates of $\hat{\rho}$ ) in all three cases. The newborn's diarrhoea also has statistically insignificant results in all cases, but blood in stool has significantly negative result in LPS only. As blood in stool has low observations in all cases, its results can be less reliable.

Table 13: Estimates of critical parameters, $\hat{\gamma}$ and $\hat{\rho}$ (IV) by state type and the whole India

\begin{tabular}{|c|c|c|c|c|c|c|c|c|c|}
\hline & \multicolumn{3}{|c|}{ High Performing State } & \multicolumn{3}{|c|}{ Low Performing State } & \multicolumn{3}{|c|}{ National } \\
\hline & Estimate & p-value & $N$ & Estimate & p-value & $N$ & Estimate & p-value & $N$ \\
\hline$\hat{\gamma}$ & $-3.153 * * *$ & $(0.008)$ & 12,768 & $-7.926 * * *$ & $(0.000)$ & 8,496 & $-5.794 * * *$ & $(0.000)$ & 18,024 \\
\hline \multicolumn{10}{|c|}{ Post-discharge complications of mother $(\widehat{\rho}(I V))$} \\
\hline High fever & -0.072 & $(0.110)$ & 12,768 & -0.024 & $(0.262)$ & 8,496 & 0.004 & $(0.804)$ & 18,024 \\
\hline Excessive bleeding & -0.002 & $(0.965)$ & 12,768 & 0.020 & $(0.364)$ & 8,496 & 0.028 & $(0.171)$ & 18,024 \\
\hline \multicolumn{10}{|c|}{ Post-discharge complications of newborn $(\widehat{\rho}(I V))$} \\
\hline Diarrhoea & 0.056 & $(0.166)$ & 12,530 & -0.007 & $(0.734)$ & 8,249 & 0.010 & $(0.508)$ & 17,634 \\
\hline Blood in stool & -0.029 & $(0.844)$ & 968 & $-0.118 * *$ & $(0.018)$ & 983 & 0.071 & $(0.299)$ & 1,345 \\
\hline
\end{tabular}

\section{Conclusion}

We explore the causal relationships between length of hospital stay and 10 post-discharge complications of mother and newborn using a large survey data on India. We argue that the main cause of inconclusive findings in the literature is due to the endogeneity of PHS. Factors including iatrogenic infection, quality of care both in hospitals (mainly for developing countries) and in a home, the intensity of delivery time complications, and socioeconomic characteristics (e.g., household income, sex of the newborn, dowry, birth order) can influence both PHS and PDCs. These can bias the true causal effects of PHS on PDCs. Our comparative analysis shows that the extent of bias can be so high that it can even turn the negative effect to a positive one.

Using the primary sample, we estimate the associations first (i.e., marginal effects of PHS on probabilities of PDCs after running separate logit regressions of dummies for PDCs on PHS and the control variables). We find almost zero but statistically significant positive associations between PHS and all available PDCs of both mother and baby. This seems implausible as we

\footnotetext{
11 It should be noted that, in Table (13), sample sizes under national category are not equal to the sums of sample sizes in HPS and LPS, as we have estimated propensity scores with different samples.
} 
expect the probabilities of PDCs to decline with increased PHS. We then utilize the dummy for JSY cash incentives as an instrument for PHS to control for the endogeneity. The nationwide program creates excess demand for institutional deliveries given the limited supply of partner hospitals' infrastructure. The treatment group thus faces the constraint of shorter hospital stay compared to the control group, the non-beneficiaries of JSY. We run two stage IV regressions, where the first stage is an OLS regression of the endogenous variable, PHS, on the instrument, JSY, and the second stage is a logit regression of the outcome variables, PDCs, on the first stage predicted value. Both the first and the second stage regressions include a set of control variables. All causal effect estimates from the second stage logit regressions (i.e., marginal effects of the predicted value of PHS on the probabilities of PDCs) are found negatives except the case of poor breastfeeding to the newborn, and most of them (e.g., 7 out of 10 PDCs) are statistically significant.

However, our primary results using IV still suffer from biases, because women are not randomly distributed between treatment and control groups of JSY: means of the control variables are different between these two groups. To establish randomization, we drop some observations by restricting the propensity score of JSY in a range on an ad hoc basis. We estimate the propensity score from a logit regression of JSY dummy on the control variables. We further drop some observations to keep those women whose first births are also the first births in their households. This ensures that the stay gap between the treatment and the control groups is not due to the mother having older children at home to take care of and thereby forcing short stay for birth order greater than 1 (first birth of a woman). This also ensures that the difference in a PDC is not due to having recently experienced member of the household (first birth in the household). The selected subsample finally shows that the differences in means of the control variables between treatment and control groups of JSY become statistically insignificant in almost all cases. The final subsample thus gives an improved validity of the instrument.

We again run two stage IV regressions in the same way as mentioned above. We find all results with negative signs, but now five PDCs (out of ten), including abdominal pain, vaginal discharge, convulsion and severe headache of a mother, and fever or cold of a baby, show statistically significant results. We find that PHS does not have significant causal effects on high fever and excessive bleeding of a mother, and poor breastfeeding, difficulty breathing, and blood in the stool of a baby.

As we do not have data on readmissions, our results are not directly comparable with those in the previous studies, but our final IV results have the same (negative) signs as found in the earlier studies. We establish negative causal effects of PHS on PDCs, which imply the positive impact of the early discharge on readmission, studied by most of the previous literature. We can compare our results, to some extent, to prior studies' findings. Studies with IV regressions will have close results to ours. For example, using an hour of birth and method of delivery as instruments in the IV regression, [10] found that the probability of readmission of the newborn in the Washington state, USA, decreases by 0.6 percentage points for 12 hours increase in PHS. If we consider the linear relationship, for one day increase in PHS, the reduction in the readmission rate will be 1.2 percentage points. In our study, we find 6.3 percentage points reduction in the probability of fever or cold of the newborn for one day increase in PHS. As the previous studies found that jaundice is the main reason for readmission of the newborn, it is unlikely that all newborns with fever or cold will be readmitted in the hospital. If we had readmission data, our result would have been pretty close to their results.

Overall, our results have moderate sizes (neither very high nor low) and are mostly sensible. For example, we see the most top negative effect of PHS on the probability of abdominal 
pain of a mother (-0.067 for one day increase in PHS). This complication can be identified in the early stage, and any measure can also be taken at the time of PHS, and a higher PHS can significantly reduce the chance of it. On the other hand, high fever and excessive bleeding of a mother and painful breathing and blood in the stool of a baby can happen at any time of the postpartum period, and rare actions can be taken at the time of PHS to prevent them, and stay gap might not influence them. We also see surprisingly no statistically significant effect of PHS on poor breastfeeding to a child, as opposed to the general view that women will learn how to breastfeed at the time of PHS and a longer PHS will reduce the chance of inadequate breastfeeding. The possible argument might be that women in developing countries mostly live in joint families where mature senior women can teach them breastfeeding, and without even staying in a hospital, they can breastfeed correctly.

We check to what extent our final results are robust by performing two identification tests - the joint randomization test and the falsification test. In the former case, we run seemingly unrelated regressions of the control variables on the JSY dummy and test whether the coefficients of the JSY dummy are jointly zero. This null hypothesis is rejected at $10 \%$ level but not rejected at 5\% level. In other words, the control variables are randomly distributed at 5\% level. Although this weak randomization is supposed to be a source of biases in our results, adding the control variables in our IV regressions controls for such biases. In the case of falsification test, we check whether PHS affects the pregnancy time morbidities. For robust results, we expect no effects of PHS on those morbidities. Our such expectation holds for ten morbidities out of thirteen. These test results imply that our final results are mostly robust.

We also estimate heterogeneous effects. The causal effects of PHS on PDCs are not linear. Higher significant negative effects are found among the women with hours of PHS is less than 72. Except for a severe headache, no effects are seen among women stayed in hospitals more than or equal to 72 hours after births. So, the negative relationships between the probabilities of PDCs and PHS are convex to the origin. We also find significant negative effects among nonscheduled caste, nontribe and nonpoor women in higher cases than their counterparts - schedule caste, tribe, and poor women. The former group of women mostly go to top public hospitals or private hospitals, and there they receive better care. A stay difference there makes significant differences in PDCs. In contrast, the latter group usually go to the bottom public hospitals where the quality of care is not of the appropriate standard and staying there is unlikely to reduce the chances of PDCs. So, the policymakers should increase not only PHS but also the quality of care in hospitals.

We also produce results for natural groups, Scheduled Caste, Tribe and Poor, without dropping observations based on propensity scores. By assuming balanced covariates between JSY and nonJSY women, we expected unbiased results in each group. However, covariates are found not balanced between JSY and nonJSY women, and thus, the second identification condition does not hold, and the results in each group are biased. Moreover, we produce results from NFHS-4, a nationally surveyed cross-section data. Our findings remain same also in low performing states and thereby in the whole of India. The results from NFHS-4 indicate the further robustness of our main findings from DLHS-4.

In conclusion, this study estimated causal effects identifies some evidence that shorter postnatal hospital stay of mother and the newborn leads to some post-discharge complications of both mother and the newborn, such as abdominal pain, vaginal discharge, convulsion and severe headache of a mother, and fever of the infant. The policy implication is that the government should facilitate sufficiently longer postnatal hospital stay of mother and her newborn, by increasing facilities and decreasing the cost of delivery. The government should also ensure the quality of 
care; otherwise, the more extended stay can make post-discharge complications up rather than low. 


\section{References}

[1] Oona MR Campbell, Luca Cegolon, David Macleod, and Lenka Benova. Length of stay after childbirth in 92 countries and associated factors in 30 low-and middle-income countries: compilation of reported data and a cross-sectional analysis from nationally representative surveys. PLoS medicine, 13(3):e1001972, 2016.

[2] Oona MR Campbell, Wendy J Graham, Lancet Maternal Survival Series steering group, et al. Strategies for reducing maternal mortality: getting on with what works. The lancet, 368(9543):1284-1299, 2006.

[3] Richard K Crump, V Joseph Hotz, Guido W Imbens, and Oscar A Mitnik. Dealing with limited overlap in estimation of average treatment effects. Biometrika, pages 1-13, 2009.

[4] William N Evans, Craig Garthwaite, Heng Wei, et al. The impact of early discharge laws on the health of newborns. Journal of health economics, 27(4):843-870, 2008.

[5] Rawad Farhat, Mariam Rajab, et al. Length of postnatal hospital stay in healthy newborns and re-hospitalization following early discharge. North American journal of medical sciences, 3(3):146, 2011.

[6] Piyush Gupta, Saurabh Malhotra, Dharmendra K Singh, and Tarun Dua. Length of postnatal stay in healthy newborns and re-hospitalization following their early discharge. The Indian Journal of Pediatrics, 73(10):897-900, 2006.

[7] K. Harron, R. Gilbert, D. Cromwell, S. Oddie, and J. van der Meulen. Newborn length of stay and risk of readmission. Paediatric and Perinatal Epidemiology, 31(3):221$232,2017$.

[8] David S Lee and Thomas Lemieuxa. Regression discontinuity designs in economics. Journal of economic literature, 48(2):281-355, 2010.

[9] Lenna L Liu, Conrad J Clemens, David K Shay, Robert L Davis, and Alvin H Novack. The safety of newborn early discharge: the washington state experience. JAMA, 278(4):293-298, 1997. 
[10] Jesse D Malkin, Michael S Broder, and Emmett Keeler. Do longer postpartum stays reduce newborn readmissions? analysis using instrumental variables. Health services research, 35(5 Pt 2):1071-1091, 2000a.

[11] Jesse D Malkin, Steven Garber, Michael S Broder, and Emmett Keeler. Infant mortality and early postpartum discharge. Obstetrics \& Gynecology, 96(2):183-188, 2000b.

[12] MoHFW. Annual report 2016-2017: Ministry of health and family welfare. Government of India, New Delhi, India, 2017.

[13] WHO. WHO recommendations on Postnatal care of the mother and newborn. World Health Organization, 2013. 


\section{8 : Public Healthcare System in India}

India's current public healthcare system is developed by following the recommendation provided in the Health Survey and Development Committee report in 1946. The goal of the recommended three-tiered system (namely primary, secondary and tertiary level of healthcare) is to provide preventive and curative health care to all rural and urban people disregarding their socioeconomic conditions and minimizing the requirement of private healthcare facilities. However, the capacity of public health care system has been insufficient to provide access to the quality care. The private health care system has got room to grow simultaneously with a constant and gradual expansion of its services. Private healthcare facilities are concentrated mostly in the urban area, but most of the JSY recipients live in the rural area and give births in public health care facilities (we show it in the next subsection). To understand why JSY recipients stay shorter in the hospital after deliveries, we need to understand the public health care system in India, which is briefly described below.

\section{Sub-health Center (SHC)}

A sub-health center (SHC) is the most peripheral public health facility at the village level, which works as the first contact point for the community people. It covers at least 5,000 people in a plain land but 3,000 in a hilly/difficult area. At least one ANM/female health worker and one male health worker are required to be staffed on a permanent basis in Each SHC. An additional ANM can also be appointed on a contract basis. It provides services for maternal and child health, family welfare, nutrition, immunization, diarrhea control and control of communicable diseases. It also provides the basic service of institutional delivery, but it cannot offer a longer postnatal stay to a woman because of no bedding facility. The Ministry of Health \& Family Welfare provides the full support to SHCs in the form of salaries, rents, and contingencies in addition to drugs and equipment. Currently, there are 148,366 SHCs in India.

\section{Primary Health Center (PHC)}

At the panchayat level, a primary health center (PHC) covers around 30,000 people in a plain area and approximately 20,000 people in a hilly/difficult area. It is the first contact point between the community people and a medical officer. Operated by at least a permanent medical officer supported by 14 paramedical staff, other staff and two additional staff nurses on a contract basis, it provides integrated curative and preventive health care services about maternal and child health, family welfare, nutrition, immunization, diarrhea control and control of communicable diseases. The State Government maintains and supports each PHC, which also provides referral services to 5-6 SHCs, with 4-6 bedding facilities for inpatients. It can accommodate women with relatively longer PHSs. Currently, 24,049 PHCs are active in India.

\section{Community Health Center (CHC)}

A community health center $(\mathrm{CHC})$ is established at the level of community development block. A plain (hilly/difficult) block has a population of around 120,000 (80,000) people. The State Government runs it with a surgeon, a physician, a gynecologist/obstetrician and a pediatrician, 
supported by 21 paramedical staff and other staff. It has around 30 beds with the facilities of an operation theater, X-ray, labor room, and laboratory. Being a referral center for PHCs and SHCs within the block, it also provides obstetric care services and specialist consultations. So, it has relatively a higher capacity of giving quality maternal and child health care services. Currently, there are 4,833 CHCs in India.

\section{Sub-district Hospital (SDH)}

A sub-district hospital (SDH), also called a sub-divisional hospital in some states, is established in the municipality area of a sub-district, along with the sub-district administrative offices. Standing as a secondary health service provider above a block level hospital, it receives referred cases from CHCs, PHCs, and SHCs in the sub-district and also in the neighboring subdistricts. It provides specialist services and acts as a first referral unit providing emergency obstetric and neonatal care services. In this way, it reduces the travel time for the emergency cases and the workload of the district hospital. It serves about 500,000-600,000 people with 30-100 or more bedding facilities. There are nearly 1,200 such hospitals operated in the country.

\section{District Hospital (DH)}

In every district, there is a district hospital (DH) located in the district headquarter town. Being the top of the district health system, it takes referral cases from SDHs, CHCs, PHCs, and SHCs in the district and provides a few tertiary healthcare services in addition to the secondary ones. There are various specialists like the surgeon, physician, obstetrician, gynecologist, pediatrician, orthopedic surgeon, ophthalmologist, anesthetist, ENT specialist and dentist work in DHs. District population varies from 30,000 to 3 million, and thus, its bedding facility ranges from 75 to 500 . Currently, there are 722 DHs in the country.

There are also a few medical colleges and specialized hospitals in large cities. These are tertiary level hospitals, which provide specialist care services in large scales. 